\title{
First-principles modeling of complexions at the phase boundaries in Ti-doped WC-Co cemented carbides at finite temperatures
}

\author{
Erik Fransson $\odot,{ }^{1, *}$ Martin Gren $\odot,{ }^{1, \dagger}$ Henrik Larsson $\odot,{ }^{2}$ and Göran Wahnström $\odot^{1, \hbar}$ \\ ${ }^{1}$ Department of Physics, Chalmers University of Technology, SE-412 96 Gothenburg, Sweden \\ ${ }^{2}$ Department of Materials Science and Engineering, Royal Institute of Technology, SE-100 44 Stockholm, Sweden
}

(Received 4 June 2021; accepted 25 August 2021; published 7 September 2021)

\begin{abstract}
WC-Co cemented carbides have a unique combination of high hardness and good toughness, making them ideal as tool materials in applications such as metal machining or rock drilling. Dopants are commonly added to retard grain growth and thereby creating a harder material. Thin films with cubic structure have been observed experimentally at phase boundaries between hexagonal WC and fcc Co-rich binder when doping with, e.g., $\mathrm{Ti}, \mathrm{V}$, or Cr. These films are generally considered to play a crucial role in the grain growth inhibition effect. Therefore, the thermodynamics of these thin cubic films is important to understand. Here, we construct, using $a b$ initio calculations and modeling, an interfacial phase diagram for thin cubic films in Ti-doped WC-Co. We consider $\mathrm{C} \leftrightarrow$ vacancy and $\mathrm{W} \leftrightarrow$ Ti substitutions by constructing alloy cluster expansions and use Monte Carlo simulations to calculate the configurational free energy. Furthermore, force-constant fitting is used to extract the harmonic free energy for the ground-state structures. Additionally, we use information from thermodynamic databases to couple our atomic-scale calculations to overall compositions of typical WC-Co materials. We predict that $\mathrm{Ti}$ segregates to WC/Co phase boundaries to form thin cubic films of two metallic layer thickness, both at solid-state and liquid-phase sintering temperatures. Furthermore, we predict that these films are stable also for low doping concentrations when no Ti-containing carbide phase precipitates in the material. We show that $\mathrm{Ti}$ essentially only segregates to the inner layer of the thin cubic film leaving an almost pure W layer towards Co, an ordering which has been observed in recent experimental high-resolution transmission electron microscopy studies.
\end{abstract}

DOI: 10.1103/PhysRevMaterials.5.093801

\section{INTRODUCTION}

For a polycrystalline material the thermodynamic and mechanical properties of its internal interfaces may be of equal importance to the macroscopic properties of the material as are the properties of the constituent bulk phases. Hence, being able to predict and understand the properties of interfaces of polycrystalline materials is important. It has been established that the appearance of certain "interfacial phases," different from those of the adjoining bulk phases (e.g., segregation structures, amorphous wetting films, etc.), can be understood from thermodynamic considerations. These disparate interfacial phases are, in fact, equilibrium states stabilized by interfacial effects and the term complexion has been suggested to separate these interfacial phases from ordinary bulk phases [1].

\footnotetext{
*erikfr@chalmers.se

†martin.gren@chalmers.se

${ }^{\ddagger}$ goran.wahnstrom@chalmers.se
}

Published by the American Physical Society under the terms of the Creative Commons Attribution 4.0 International license. Further distribution of this work must maintain attribution to the author(s) and the published article's title, journal citation, and DOI. Funded by Bibsam.
Cemented carbides, or hard metals, are a class of materials which consist of fine particles of a carbide, often $\mathrm{WC}$, cemented into a composite by a binder metal, often Co [2-4]. They combine excellent hardness with high toughness and wear resistance, making them ideal as material in cutting and wear resistant tools $[5,6]$. Cemented carbides are, therefore, of great industrial importance [7,8].

The excellent mechanical properties of cemented carbides are heavily dependent on the microstructure of the material, which in turn depends on the interfacial properties. For instance, the hardness of cemented carbides is anticorrelated with the average size of the carbide grains, i.e., smaller grains mean a harder material [9]. During the sintering of these materials the carbide grains grow by means of solution reprecipitation. To keep the carbide grain size low, the material is often doped with, e.g., $\mathrm{V}, \mathrm{Cr}$, or $\mathrm{Ti}$, which act as grain growth inhibitors giving rise to a finer microstructure and a harder material [10-13].

One reason for the grain growth inhibition effect is thought to be thin cubic films of mixed carbides forming at the interfaces between the grains of the main carbide and the binder, and thereby introducing additional kinetic barriers in the solution-reprecipitation process, similar to a solute drag effect $[14,15]$. These thin films, or complexions, have been observed experimentally at the phase boundary between the hexagonal WC and Co in, e.g., V-doped [16], Cr-doped [17], and Ti-doped [18] WC-Co systems. Moreover, in Ti-doped 
WC-Co cemented carbides the Ti atoms are found to segregate to the second metal layer of the thin cubic film, leaving a layer of $\mathrm{W}$ atoms towards Co $[18,19]$.

In this paper, we present a theoretical modeling approach of the interfacial properties of thin cubic films in doped WCCo cemented carbides at finite temperatures. We apply the approach to thin cubic films on the basal, i.e., (0001), WC interface plane in Ti-doped WC-Co cemented carbides. In a previous study thin cubic films in $\mathrm{V}$-doped cemented carbides were investigated [20]. Here, we extend that methodology and include vibrational degrees of freedom and the presence of carbon vacancies in the thin films and, in order to make the computations practicable, recently developed software techniques $[21,22]$ are used. This results in a complete firstprinciples based modeling of the formation of thin cubic films in doped cemented carbides at finite temperatures.

We consider several different types of cubic thin films with varying thickness in a Ti-doped material. For each of these we construct alloy cluster expansions (CEs) for both W-Ti and C-vacancy substitutions fitted to density functional theory (DFT) calculations using the ICET software [21]. The CEs are used to find both ground-state structures as well as the configurational free energy as a function of $\mathrm{Ti}$ and $\mathrm{C}$ concentrations. Thereafter, the vibrational free energy is calculated from the ground-state structures in the harmonic approximation (HA) using the software HIPHIVE [22]. The total free-energy landscape of the thin films is then obtained as a function of temperature and chemical potentials for $\mathrm{C}$ and Ti.

To relate this to a real material, defined by its bulk composition, i.e., the concentration of the atomic species $\mathrm{W}, \mathrm{C}, \mathrm{Co}$, and $\mathrm{Ti}$, we make use of the THERMO-CALC software [23,24]. We need to obtain the chemical potentials for $\mathrm{Ti}$ and $\mathrm{C}$ in the real W-C-Co-Ti system as function of temperature and bulk composition. This information can be used to construct an "'interfacial phase diagram," a complexion diagram, which describes for which compositions of the real material a thinfilm structure is stable as function of temperature.

We show that cubic films are thermodynamically stable both at solid state and liquid to the right a high-temperature structure from the Monte Carlo (MC) simulation at $1670 \mathrm{~K}$ is shown; here Va denotes carbon vacancies phase sintering temperatures. Further, the cubic films are shown to be thermodynamically stable also below the Ti dopant concentrations for which the gamma phase, i.e., $\gamma-(\mathrm{Ti}, \mathrm{W}) \mathrm{C}_{x}$, is stable due to favorable interfacial properties of the Ti-rich films. Moreover, our calculations confirm that Ti segregates to the second layer of the thin film leaving one $\mathrm{W}$ layer towards $\mathrm{Co}$ and we also provide an atomistic explanation as to why this ordering occurs.

\section{STRUCTURAL MODELING}

The first step in our atomic-scale approach is to define suitable interfacial model structures. Motivated by experimental findings from the literature, we follow the approach introduced in Ref. [20].

Thin cubic films have been observed in Ti-doped WC at the phase boundary between the hexagonal WC $(\delta$-WC) [25] basal plane (0001) and fcc $\mathrm{Co}$ in high-resolution transmis- sion electron microscopy (HRTEM) studies [12,18,19]. The orientation of cubic films were such that the [111] direction was aligned with the basal WC plane. Moreover, in Ref. [18] it is indicated that the [111] direction of fcc Co aligns with the [111] direction of cubic $(\mathrm{Ti}, \mathrm{W}) \mathrm{C}_{x}$. Based on this information the following orientation

$$
\delta \text {-WC (0001) }\left\|(\mathrm{Ti}, \mathrm{W}) \mathrm{C}_{x}(111)\right\| \mathrm{Co}(111)
$$

is used for the model interfaces in this study.

In the hexagonal crystal structure of $\delta$-WC layers of W and $\mathrm{C}$ are alternating and stacked in an $A B A B A B \ldots$ sequence along the basal [0001] direction. The cubic $(\mathrm{Ti}, \mathrm{W}) \mathrm{C}_{x}$ structure also has alternating layers of $(\mathrm{Ti}, \mathrm{W})$ and $\mathrm{C}$ along its [111] direction, however, the stacking sequence is $A B C A B C \ldots$. Here, $A$ corresponds to a translation (in scaled coordinates) of the atomic layer by $(0,0), B$ by $\left(\frac{1}{3}, \frac{2}{3}\right)$, and $C$ by $\left(\frac{2}{3}, \frac{1}{3}\right)$ in the $x y$ plane. Therefore, the $\delta$-WC structure can be converted to a cubic WC $(\gamma$-WC) [25] structure by altering the stacking sequence. In this way multiple different $\delta$-WC $(0001) / \gamma$ WC (111) interfaces can be constructed. Further, in the $\gamma$-WC structure $\mathrm{W}$ atoms may be switched to $\mathrm{Ti}$ and $\mathrm{C}$ vacancies may be created giving a general cubic $(\mathrm{Ti}, \mathrm{W}) \mathrm{C}_{x}$ structure. In this interface construction the $(\mathrm{Ti}, \mathrm{W}) \mathrm{C}_{x}$ phase is strained in the (111) plane such that the $(\mathrm{Ti}, \mathrm{W})$ and $\mathrm{C}$ layers along [111] matches the corresponding layers in $\delta$-WC.

The interface energy for both $\delta$-WC (0001)/Co (111) and $\gamma$-WC (111)/Co (111) is less for a W-terminated carbide plane compared with a C-terminated carbide plane [26]. Also, the TiC (111)/Co (111) interface energy is less with Ti termination compared to $C$ termination (see Supplemental Material [27]). In all cases we find that the interface energy for the metal-terminated interface is at least $1 \mathrm{~J} / \mathrm{m}^{2}$ less compared with the carbon-terminated interface. Hence, we consider a (Ti,W)-terminated carbide plane towards Co.

We allow four atomic layers of $\delta$-WC to change stacking and generate all possible stacking sequences, as done in Ref. [26]. This leads to nine possible stackings, including the regular $\delta$-WC (0001) and Co (111) phase boundary. The stacking sequences are given in Fig. 1 and the $i$ th metal and carbon layers counting from the cobalt is denoted $\mathrm{Ml}_{i}$ and $\mathrm{Cl}_{i}$, respectively.

The Co slab is oriented such that there is a $30^{\circ}$ angle between $\delta$-WC [2 $\overline{1} \overline{1} 0]$ and $\mathrm{Co}[\overline{1} 10]$ in order to get small repeating unit cells with low amount of strain in the Co phase. This gives a unit cell with four Co atoms and three $\mathrm{W}$ or $\mathrm{C}$ atoms in each atomic layer along the [111] and [0001] directions, respectively. In the unit cell there are thus three sites per $\mathrm{W}$ and $\mathrm{C}$ atomic layer.

We let $m_{\mathrm{C}}$ define the number of carbon sites which can be occupied by vacancies, and $m_{\mathrm{M}}$ the number of metal sites which can be occupied by either Ti or W. For stackings $k=1$ to 7, all sites that belong to the four outermost carbide layers are regarded as mixing sites. This leads to a total number of mixing sites $m=m_{\mathrm{C}}+m_{\mathrm{M}}=12$ in the unit cell. For stacking $k=8$ one additional metal layer $\left(\mathrm{Ml}_{3}\right)$ is regarded as mixing sites, which yields a total of $m=15$ mixing sites. In the reference system, $k=0$, no carbon vacancies or Ti substitutions are considered because this is associated with a high-energy cost. See Supplemental Material for more details on the selection of mixing sites for the various stackings [27]. 


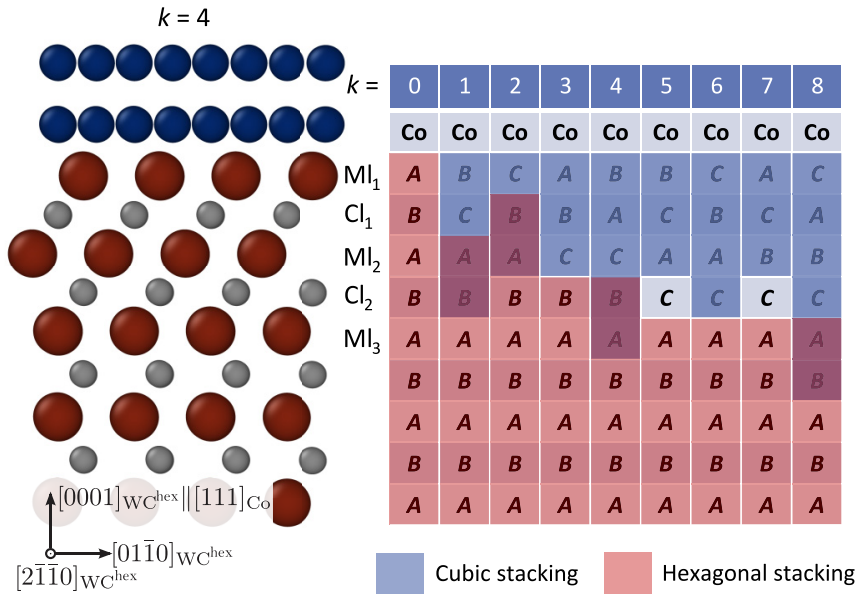

FIG. 1. Illustration of the nine different stackings. The left figure illustrates the atomic configuration at the WC/Co interface with the $k=4$ stacking (generated by OVITO [28]). C, Co, and W are represented by gray, blue, and red atoms, respectively. The right table indicates the stacking sequences for the various $k$ 's and which atomic layers follow the hexagonal stacking (red) and the cubic stacking (blue).

We denote the carbon vacancy and Ti site concentration in the film as $x_{\mathrm{Va}}$ and $x_{\mathrm{Ti}}$, respectively. The Ti concentration is defined as $x_{\mathrm{Ti}}=n_{\mathrm{Ti}} / m_{\mathrm{M}}$, where $n_{\mathrm{Ti}}$ is the total number of $\mathrm{Ti}$ atoms in the unit cell. The vacancy concentration is defined analogously, $x_{\mathrm{Va}}=1-n_{\mathrm{C}} / m_{\mathrm{C}}$, where $n_{\mathrm{C}}$ is the total number of $\mathrm{C}$ atoms in the unit cell.

\section{THERMODYNAMICS}

\section{A. Interface energy}

The interface energy $\gamma$, i.e., the cost associated with creating an interface per interface area, is in general written as

$$
\gamma(T)=\frac{1}{A}\left(G(T)-\sum_{i} N_{i} \mu_{i}(T)\right),
$$

where $G(T)$ is the Gibbs free energy for the interface system at a given temperature $T, A$ the area of the interface, and $N_{i}$ and $\mu_{i}$ are the number of atoms and chemical potential of each species, respectively [29].

We let $g_{k}\left(x_{\mathrm{Va}}, x_{\mathrm{Ti}}, T\right)$ denote the free energy of a film with stacking sequence $k$ per unit cell. Additionally, we let $a$ denote the interface area for the unit cell, i.e., $G / A=g / a$. The interface energy of a film can then be written as

$$
\begin{array}{r}
\gamma_{k}\left(x_{\mathrm{Va}}, x_{\mathrm{Ti}}, T\right)=\frac{1}{a}\left(g_{k}\left(x_{\mathrm{Va}}, x_{\mathrm{Ti}}\right)-m_{\mathrm{C}}\left(1-x_{\mathrm{Va}}\right) \mu_{\mathrm{C}}\right. \\
\left.-m_{\mathrm{M}}\left(1-x_{\mathrm{Ti}}\right) \mu_{\mathrm{W}}-m_{\mathrm{M}} x_{\mathrm{Ti}} \mu_{\mathrm{Ti}}-\sum_{i \notin \operatorname{mix}} \mu_{i}\right),
\end{array}
$$

where the temperature dependency of the right-hand side terms has been dropped for simplicity. Further, $m_{\mathrm{C}}$ and $m_{\mathrm{M}}$ are the number of mixing sites for the carbon and metal atoms, respectively, in the unit cell and the summation in the last term extends over all sites excluding the mixing sites. For $k=0$ (i.e., no thin cubic film), which is modeled without carbon vacancies $\left(x_{\mathrm{Va}}=0\right)$ and Ti substitutions $\left(x_{\mathrm{Ti}}=0\right)$, this simplifies to

$$
\gamma_{0}(T)=\frac{1}{a}\left(g_{0}-m_{\mathrm{C}} \mu_{\mathrm{C}}-m_{\mathrm{M}} \mu_{\mathrm{W}}-\sum_{i \notin \operatorname{mix}} \mu_{i}\right),
$$

where again temperature dependencies have been left out for simplicity's sake.

Rather than looking at the absolute interface energy $\gamma_{k}\left(x_{\mathrm{Va}}, x_{\mathrm{Ti}}, T\right)$, we study the relative interface energy $\Delta \gamma_{k}\left(x_{\mathrm{Va}}, x_{\mathrm{Ti}}, T\right)$, defined as

$$
\Delta \gamma_{k}\left(x_{\mathrm{Va}}, x_{\mathrm{Ti}}, T\right)=\gamma_{k}\left(x_{\mathrm{Va}}, x_{\mathrm{Ti}}, T\right)-\gamma_{0}(T) .
$$

The relative interface energy is therefore a measure of the tendency to create a thin cubic film structure and this is the key quantity in this study. If $\Delta \gamma$ is negative, film formation is favorable, otherwise it is unfavorable. From Eqs. (1) and (2) we see that in the expression for $\Delta \gamma$ the chemical potentials of nonmixing sites cancel out. We thus obtain

$$
\Delta \gamma_{k}=\frac{1}{a}\left(g_{k}-g_{0}+m_{\mathrm{C}} x_{\mathrm{Va}} \mu_{\mathrm{C}}+m_{\mathrm{M}} x_{\mathrm{Ti}}\left(\mu_{\mathrm{W}}-\mu_{\mathrm{Ti}}\right)\right),
$$

where all $\left(x_{\mathrm{Va}}, x_{\mathrm{Ti}}, T\right)$ dependencies have been dropped for simplicity.

\section{B. Chemical potentials}

As reference states for the chemical potentials we use stoichiometric hexagonal WC, graphite, and stoichiometric TiC in equilibrium with each other. Deviations from this combination of reference states are described by $\Delta \mu$ terms. The chemical potential for atom $i$ is thus decomposed as

$$
\mu_{\mathrm{i}}(T)=\mu_{\mathrm{i}}^{\mathrm{ref}}(T)+\Delta \mu_{\mathrm{i}},
$$

where $\mu_{\mathrm{i}}^{\text {ref }}(T)$ is the chemical potential for atom $i$ in the chosen set of reference states.

The carbon chemical potential is then given by

$$
\mu_{\mathrm{C}}(T)=g_{\text {gra }}(T)+\Delta \mu_{\mathrm{C}},
$$

where $g_{\text {gra }}(T)=g_{\text {gra }}^{0}+g_{\text {gra }}^{\text {vib }}(T)$ is the energy and vibrational contribution to the free energy of graphite. The titanium chemical potential is given by

$$
\mu_{\mathrm{Ti}}(T)=g_{\mathrm{TiC}}(T)-g_{\text {gra }}(T)+\Delta \mu_{\mathrm{Ti}},
$$

where $g_{\mathrm{TiC}}(T)=g_{\mathrm{TiC}}^{0}+g_{\mathrm{TiC}}^{\mathrm{vib}}(T)$ is the energy and vibrational contribution to the free energy of the stoichiometric TiC bulk phase per TiC unit. Further, we assume that the real material, considered in this work, is always in equilibrium with stoichiometric hexagonal WC and, hence,

$$
\mu_{\mathrm{W}}(T)+\mu_{\mathrm{C}}(T)=g_{\mathrm{WC}}(T),
$$

where $g_{\mathrm{WC}}(T)=g_{\mathrm{WC}}^{0}+g_{\mathrm{WC}}^{\mathrm{vib}}(T)$ is the energy and vibrational contribution to the free energy of the stoichiometric WC bulk phase per WC unit. The assumption of equilibrium with stoichiometric $\delta$-WC, also at high temperatures, is very well founded [30]. The chemical potential for tungsten is thus given by

$$
\mu_{\mathrm{W}}(T)=g_{\mathrm{WC}}(T)-g_{\text {gra }}(T)-\Delta \mu_{\mathrm{C}}
$$


and, therefore, $\Delta \mu_{\mathrm{W}}=-\Delta \mu_{\mathrm{C}}$. The cobalt chemical potential, while relevant for absolute interface energies, becomes irrelevant for understanding the thermodynamic stability of the cubic films [cf. Eq. (4)].

Only two of the $\Delta \mu$ terms are independent, here chosen to be $\Delta \mu_{\mathrm{C}}$ and $\Delta \mu_{\mathrm{Ti}}$. We denote the change in chemical potential due to these terms an environmental effect.

\section{Free-energy decomposition}

The free energy of a film $g_{k}\left(x_{\mathrm{Va}}, x_{\mathrm{Ti}}, T\right)$ can be decomposed as

$$
\begin{aligned}
g_{k}\left(x_{\mathrm{Va}}, x_{\mathrm{Ti}}, T\right)= & g_{k}^{0}\left(x_{\mathrm{Va}}, x_{\mathrm{Ti}}\right)+g_{k}^{\mathrm{conf}}\left(x_{\mathrm{Va}}, x_{\mathrm{Ti}}, T\right) \\
& +g_{k}^{\mathrm{vib}}\left(x_{\mathrm{Va}}, x_{\mathrm{Ti}}, T\right),
\end{aligned}
$$

where $g_{k}^{0}\left(x_{\mathrm{Va}}, x_{\mathrm{Ti}}\right)$ is the energy, $g_{k}^{\text {conf }}\left(x_{\mathrm{Va}}, x_{\mathrm{Ti}}, T\right)$ is the configurational free energy, and $g_{k}^{\mathrm{vib}}\left(x_{\mathrm{Va}}, x_{\mathrm{Ti}}, T\right)$ the vibrational free energy. For the $k=0$ case the decomposition becomes

$$
g_{0}(T)=g_{0}^{0}+g_{0}^{\mathrm{vib}}(T)
$$

since there are no substitutions and hence no configurational contribution to the free energy.

Next, the free energy, Eqs. (8) and (9), and the chemical potentials, Eqs. (5) and (6), are inserted into the expression for the relative interface energy, Eq. (4). This allows for a natural decomposition of the relative interface energy into four parts as

$$
\begin{aligned}
& \Delta \gamma_{k}\left(x_{\mathrm{Va}}, x_{\mathrm{Ti}}, T\right)=\Delta \gamma_{k}^{0}\left(x_{\mathrm{Va}}, x_{\mathrm{Ti}}\right)+\Delta \gamma_{k}^{\mathrm{conf}}\left(x_{\mathrm{Va}}, x_{\mathrm{Ti}}, T\right) \\
& +\Delta \gamma_{k}^{\mathrm{vib}}\left(x_{\mathrm{Va}}, x_{\mathrm{Ti}}, T\right)+\Delta \gamma_{k}^{\text {env }}\left(x_{\mathrm{Va}}, x_{\mathrm{Ti}}\right)
\end{aligned}
$$

corresponding to energetic, configurational, vibrational, and environmental contributions, respectively. The energetic part is given by

$$
\begin{aligned}
\Delta \gamma_{k}^{0}\left(x_{\mathrm{Va}}, x_{\mathrm{Ti}}\right)= & \frac{1}{a}\left[g_{k}^{0}\left(x_{\mathrm{Va}}, x_{\mathrm{Ti}}\right)-g_{0}^{0}\right. \\
& \left.+m_{\mathrm{C}} x_{\mathrm{Va}} g_{\mathrm{gra}}^{0}+m_{\mathrm{M}} x_{\mathrm{Ti}}\left(g_{\mathrm{WC}}^{0}-g_{\mathrm{TiC}}^{0}\right)\right] .
\end{aligned}
$$

The configurational part of the relative interface free energy becomes

$$
\Delta \gamma_{k}^{\mathrm{conf}}\left(x_{\mathrm{Va}}, x_{\mathrm{Ti}}, T\right)=\frac{1}{a} g_{k}^{\mathrm{conf}}\left(x_{\mathrm{Va}}, x_{\mathrm{Ti}}, T\right)
$$

since the film is the only system for which substitutions is considered. The vibrational part, analogous to energetic part, becomes

$$
\begin{aligned}
\Delta \gamma_{k}^{\mathrm{vib}}\left(x_{\mathrm{Va}}, x_{\mathrm{Ti}}, T\right) & \\
= & \frac{1}{a}\left[g_{k}^{\mathrm{vib}}\left(x_{\mathrm{Va}}, x_{\mathrm{Ti}}, T\right)-g_{0}^{\mathrm{vib}}(T)\right. \\
& \left.\quad+m_{\mathrm{C}} x_{\mathrm{Va}} g_{\mathrm{gra}}^{\mathrm{vib}}(T)+m_{\mathrm{M}} x_{\mathrm{Ti}}\left(g_{\mathrm{WC}}^{\mathrm{vib}}(T)-g_{\mathrm{TiC}}^{\mathrm{vib}}(T)\right)\right] .
\end{aligned}
$$

Finally, the environmental part becomes

$$
\Delta \gamma_{k}^{\text {env }}\left(x_{\mathrm{Va}}, x_{\mathrm{Ti}}\right)=\frac{1}{a}\left[m_{\mathrm{C}} x_{\mathrm{Va}} \Delta \mu_{\mathrm{C}}-m_{\mathrm{M}} x_{\mathrm{Ti}}\left(\Delta \mu_{\mathrm{Ti}}+\Delta \mu_{\mathrm{C}}\right)\right],
$$

which is the only part that depends on $\Delta \mu_{\mathrm{C}}$ and $\Delta \mu_{\mathrm{Ti}}$.

\section{Equilibrium stacking}

The last step is to transform the relative interface energy from a function of $\left(x_{\mathrm{Va}}, x_{\mathrm{Ti}}\right)$ to a function of $\left(\Delta \mu_{\mathrm{C}}, \Delta \mu_{\mathrm{Ti}}\right)$ via

$\Delta \gamma_{k}\left(\Delta \mu_{\mathrm{C}}, \Delta \mu_{\mathrm{Ti}}, T\right)=\min _{x_{\mathrm{Va}}, x_{\mathrm{Ti}}}\left\{\Delta \gamma_{k}\left(x_{\mathrm{Va}}, x_{\mathrm{Ti}}, T\right): \Delta \mu_{\mathrm{C}}, \Delta \mu_{\mathrm{Ti}}\right\}$,

where minimization should be read as the minimum of $\Delta \gamma_{k}\left(x_{\mathrm{Va}}, x_{\mathrm{Ti}}, T\right)$ with respect to both $x_{\mathrm{Va}}$ and $x_{\mathrm{Ti}}$ given $\Delta \mu_{\mathrm{C}}$ and $\Delta \mu_{\mathrm{Ti}}$.

The equilibrium stacking is obtained by whichever stacking $k$ that minimizes $\Delta \gamma_{k}\left(\Delta \mu_{\mathrm{C}}, \Delta \mu_{\mathrm{Ti}}, T\right)$. If no stacking $k$ has a negative value of $\Delta \gamma_{k}\left(\Delta \mu_{\mathrm{C}}, \Delta \mu_{\mathrm{Ti}}, T\right)$, then $k=0$ (i.e., no thin cubic film) is thermodynamically stable.

\section{COMPUTATIONAL METHODS}

The modeling in this study is based on electronic structure calculations using DFT as implemented in the Vienna $a b$ initio simulation package (VASP) [31,32]. For more details see Appendix A. The electronic structure calculations are used to determine ground-state properties and the models employed for describing configurational and vibrational effects require extensive electronic structure based reference data to be trained on.

However, the chemical potential differences $\Delta \mu_{\mathrm{C}}$ and $\Delta \mu_{\mathrm{Ti}}$ between well-defined reference states and a real $\mathrm{W}-\mathrm{C}$ $\mathrm{Co}$-Ti material at finite temperatures are difficult to obtain from DFT calculations. Therefore, we make use of experimental data, by means of thermodynamic calculations using CALPHAD [33] databases, to obtain these chemical potential differences. For more details see Appendix B.

\section{A. Configurational degrees of freedom}

Alloy CEs are employed in order to study the mixing of carbon atoms and vacancies as well as tungsten and titanium in the films. For convenience we introduce a mixing free energy per mixing site $m, g_{k}^{\operatorname{mix}}\left(x_{\mathrm{Va}}, x_{\mathrm{Ti}}, T\right)$, defined as

$$
\begin{array}{r}
m g_{k}^{\operatorname{mix}}\left(x_{\mathrm{Va}}, x_{\mathrm{Ti}}, T\right)=g_{k}^{0}\left(x_{\mathrm{Va}}, x_{\mathrm{Ti}}\right)+g_{k}^{\mathrm{conf}}\left(x_{\mathrm{Va}}, x_{\mathrm{Ti}}, T\right) \\
-g_{k}^{0}(0,0)+m_{\mathrm{C}} x_{\mathrm{Va}} g_{\mathrm{gra}}^{0}+m_{\mathrm{M}} x_{\mathrm{Ti}}\left(g_{\mathrm{WC}}^{0}-g_{\mathrm{TiC}}^{0}\right),
\end{array}
$$

which thus expresses the deviation in free energy of the film from $g_{k}^{0}(0,0)$ and the corresponding references. The mixing free energy $g_{k}^{\operatorname{mix}}\left(x_{\mathrm{Va}}, x_{\mathrm{Ti}}, T\right)$ can be written as

$$
g^{\operatorname{mix}}(T)=e^{\operatorname{mix}}(T)-T s^{\operatorname{mix}}(T),
$$

where the stacking $(k)$ and concentration $(x)$ dependencies have been dropped for simplicity. Here, $e^{\operatorname{mix}}$ is the mixing energy and $s^{\text {mix }}$ the mixing entropy.

The mixing energy is modeled via CEs using the software ICET [21]. The CE models allow for very fast predictions of the mixing energy of different configurations. This makes it feasible to search for low-energy structures as well as sample the configurational free energy via MC simulations [34].

To obtain the configurational free energy we use the canonical ensemble. The mixing energy is obtained from

$$
e^{\operatorname{mix}}(T)=\frac{1}{N_{\mathrm{MC}}}\left\langle E_{\mathrm{MC}}(T)\right\rangle,
$$


where $\langle\ldots\rangle$ denotes an ensemble average which is evaluated using $\mathrm{MC}$ simulations and $E_{\mathrm{MC}}$ is the total mixing energy for a supercell with $N_{\mathrm{MC}}$ mixing sites. In the MC simulations we use $N_{\mathrm{MC}}=432$.

To determine the mixing entropy we first evaluate the heat capacity per mixing site according to

$$
c_{V}(T)=\frac{1}{N_{\mathrm{MC}}} \frac{\left\langle E_{\mathrm{MC}}(T)^{2}\right\rangle-\left\langle E_{\mathrm{MC}}(T)\right\rangle^{2}}{k_{\mathrm{B}} T^{2}} .
$$

The mixing entropy is then obtained, using the thermodynamic relation $\partial s / \partial T=c_{V} / T$, by integration

$$
s^{\operatorname{mix}}(T)=s^{\operatorname{mix}}(\infty)+\int_{\infty}^{T} \frac{c_{V}\left(T^{\prime}\right)}{T^{\prime}} d T^{\prime},
$$

where $s^{\text {mix }}(\infty)$ is known as the ideal mixing. In our case with two sublattices of size $m_{\mathrm{M}}$ and $m_{\mathrm{C}}$, respectively, we have an ideal mixing entropy of

$$
s^{\text {mix }}(\infty)=\frac{m_{\mathrm{M}}}{m} s_{\text {ideal }}\left(x_{\mathrm{Ti}}\right)+\frac{m_{\mathrm{C}}}{m} s_{\text {ideal }}\left(x_{\mathrm{Va}}\right),
$$

where

$$
s_{\text {ideal }}(x)=-k_{\mathrm{B}}[x \ln (x)+(1-x) \ln (1-x)] .
$$

Finally, once $e^{\operatorname{mix}}(T)$ and $s^{\operatorname{mix}}(T)$ are obtained, the mixing free energy $g^{\text {mix }}(T)$ can be obtained via Eq. (16).

In Fig. 2 an example of this temperature integration is shown for $k=4$ and for two cases: (i) $x_{\mathrm{Ti}}=0.5$ and $x_{\mathrm{Va}}=0.0$, and (ii) $x_{\mathrm{Ti}}=0.5$ and $x_{\mathrm{Va}}=0.167$. For low temperatures the heat capacity and entropy go toward zero as the ground-state structure starts to dominate, making also the difference between the energy and free energy approach zero. For high temperatures the entropies approach the ideal mixing limit. The system with more available states $\left(x_{\mathrm{Ti}}=0.5\right.$ and $x_{\mathrm{Va}}=$ 0.167 ) has a larger entropy and thus obtains a lower free energy as temperature is increased.

\section{B. Vibrational degrees of freedom}

The vibrational free energy is calculated using the harmonic approximation, where the harmonic frequencies $\omega_{i}$ are obtained from the second-order force constants $\Phi_{i j}$. The harmonic free energy can be calculated via

$$
F^{\mathrm{vib}}=\sum_{i}\left\{\frac{\hbar \omega_{i}}{2}+k_{\mathrm{B}} T \ln \left[1-\exp \left(-\frac{\hbar \omega_{i}}{k_{\mathrm{B}} T}\right)\right]\right\},
$$

where the summation over $i$ goes over all frequencies in the system [35].

The second-order force constants $\Phi_{i j}$ are constructed for the ground-state structures for a range of both vacancy and $\mathrm{Ti}$ concentrations. Because of the large primitive cell and low symmetry of the structures, we employ the regression approach to extract the harmonic force constants using HIPHIVE $[22,36]$. The displacements of the training structures are generated from a normal distribution with a standard deviation of $0.01 \AA$. The summation in Eq. (22) is carried out using PHONOPY [37].

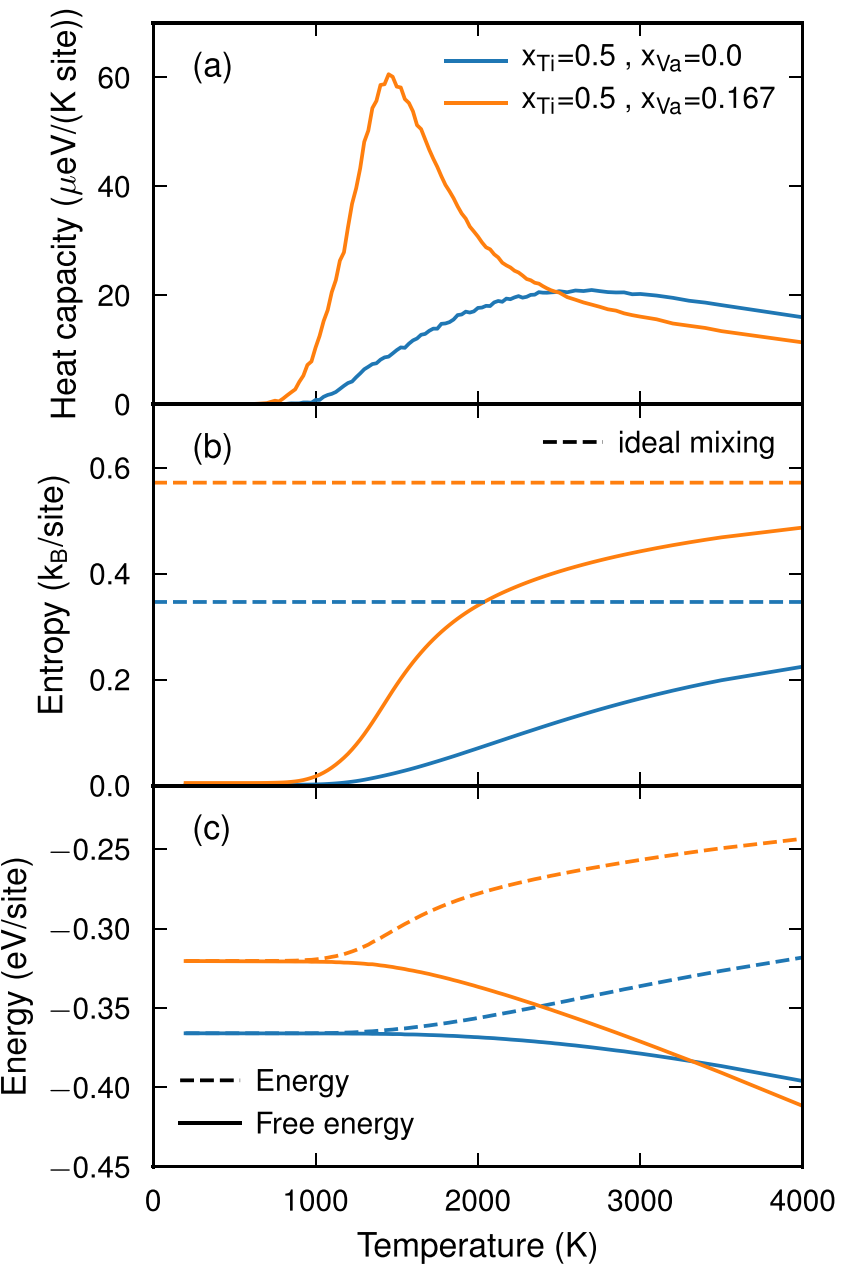

FIG. 2. Demonstration of configurational free-energy integration for $k=4$ and for two cases: (i) $x_{\mathrm{Ti}}=0.5$ and $x_{\mathrm{Va}}=0.0$, and (ii) $x_{\mathrm{Ti}}=0.5$ and $x_{\mathrm{Va}}=0.167$. (a) Heat capacity as a function of temperature. (b) Mixing entropy as a function of temperature where dashed lines indicate the ideal mixing limits. (c) Energy and free energy as a function of temperature.

\section{Chemical potentials}

To obtain the chemical potentials in Eqs. (5) and (6) we first determine $g^{0}$ and $g^{\mathrm{vib}}(T)$ for the three different phases: graphite, stoichiometric TiC, and hexagonal WC using DFT. For graphite the DFT calculations are done for the diamond phase, due to inaccuracies of the PBE approximation for graphite. We then use the experimental shift for the free energy between diamond and graphite at $300 \mathrm{~K}$ to obtain the chemical potential for graphite as done in Ref. [38].

The next step is to determine $\Delta \mu_{\mathrm{C}}$ and $\Delta \mu_{\mathrm{Ti}}$. This is done using experimental input. We consider a W-C-Co-Ti system and make use of the software THERMO-CALC [23]. For details, see Appendix B.

In Fig. 3 we show the result for $\Delta \mu_{\mathrm{C}}$ (left panel) and $\Delta \mu_{\mathrm{Ti}}$ (right panel) at $1670 \mathrm{~K}$. This temperature is a typical liquidphase sintering temperature for $\mathrm{WC} / \mathrm{Co}$. We vary the atomic concentrations of $\mathrm{C}$ and $\mathrm{Ti}$ in the ranges of $40<c_{\mathrm{C}}<44$ at.\% and $0<c_{\mathrm{Ti}}<0.6$ at. $\%$, respectively.

Consider the left panel. We note that $\Delta \mu_{\mathrm{C}}$ becomes zero for $c_{\mathrm{C}}$ larger than about 43.0 at.\%, corresponding to graphite 

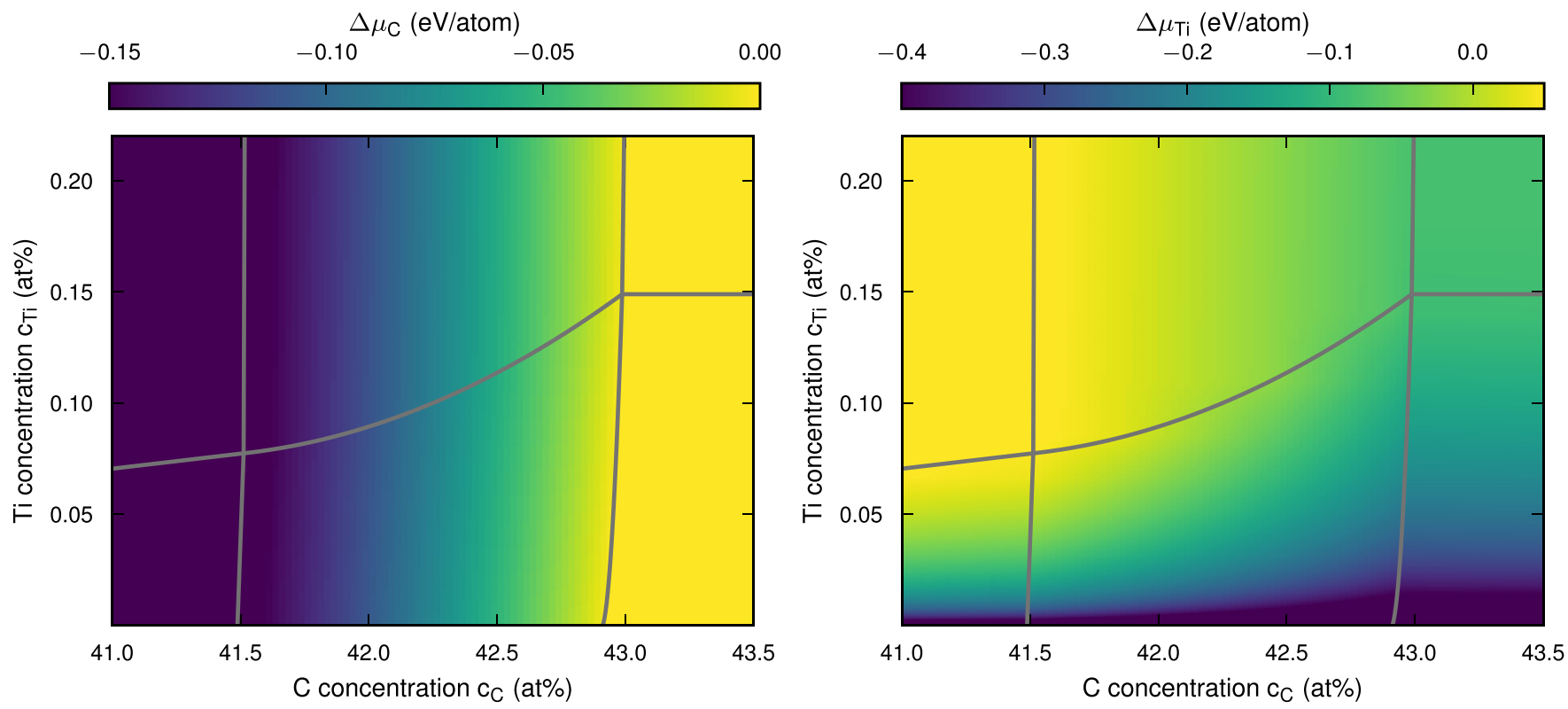

FIG. 3. Heat maps for the chemical potential differences $\Delta \mu_{\mathrm{C}}$ (left) and $\Delta \mu_{\mathrm{Ti}}$ (right) as a function of atomic concentrations (at.\%) of carbon $c_{\mathrm{C}}$ and titanium $c_{\mathrm{Ti}}$ at $1670 \mathrm{~K}$. The gray vertical lines indicate the phase boundary regions where graphite (rightmost region) and eta (leftmost region) is stable. The middle region is often referred to as the carbon window. The gray horizontal line indicates the phase boundary where $\gamma$ - $(\mathrm{Ti}, \mathrm{W}) \mathrm{C}_{x}$ becomes stable. Here, $\Delta \mu_{\mathrm{Ti}}$ values lower than $-0.4 \mathrm{eV} /$ atom are shown as $-0.4 \mathrm{eV} /$ atom . The $\mathrm{Co}$ to $\mathrm{W}$ atomic ratio is locked to $c_{\mathrm{Co}} / c_{\mathrm{W}}=0.373$.

precipitating at these concentrations. For $c_{\mathrm{C}}$ less than about 41.5 at. $\%, \Delta \mu_{\mathrm{C}}$ becomes constant and about $-0.15 \mathrm{eV}$, corresponding to precipitation of the eta phase, a cobalt-containing carbide phase. These two limits makes up the range for industrially allowed values of $\mu_{\mathrm{C}}$ since eta and graphite are undesirable phases in cemented carbides. This range corresponds to the "carbon window" within which tungsten carbide WC and a Co-rich melt are stable.

Consider now the right panel. For $c_{\mathrm{Ti}}$ lower than about 0.1 at.\% $\mathrm{Ti}$ is dissolved in the Co liquid, while at higher values for $c_{\mathrm{Ti}}$ the $\gamma-(\mathrm{Ti}, \mathrm{W}) \mathrm{C}_{x}$ phase becomes stable. The Ti chemical potential $\Delta \mu_{\mathrm{Ti}}$ increases with increasing Ti concentration until $\gamma$-(Ti, W) $\mathrm{C}_{x}$ become stable after which a constant value is obtained. In the graphite limit $\left(c_{\mathrm{C}}=43.0\right.$ at.\% $), \gamma$-(Ti, W $) \mathrm{C}_{x}$ becomes stable at $c_{\mathrm{Ti}}=0.15$ at.\%, for which the chemical potential is $\Delta \mu_{\mathrm{Ti}}=-0.088 \mathrm{eV}$. In the eta limit $\gamma-(\mathrm{Ti}, \mathrm{W}) \mathrm{C}_{x}$ becomes stable at $c_{\mathrm{Ti}}=0.077$ at. $\%$ with $\Delta \mu_{\mathrm{Ti}}=0.056 \mathrm{eV}$.

The chemical potentials at $1300 \mathrm{~K}$ (corresponding to solidstate sintering temperature) are shown in the Supplemental Material (Fig. S4) [27].

\section{RESULTS}

\section{A. Ground-state contribution}

First we consider the relative interface energy at $0 \mathrm{~K}$, $\Delta \gamma_{k}^{0}\left(x_{\mathrm{Va}}, x_{\mathrm{Ti}}\right)$, defined in Eq. (11). To restrict the number of stackings $k$ to be investigated we calculated the change in the interface energy due to $\mathrm{W} \rightarrow$ Ti substitutions in the outermost four metal layers with DFT (see Supplemental Material [27]). $\mathrm{W} \rightarrow \mathrm{Ti}$ substitutions are particularly favorable in $\mathrm{Ml}_{2}$ of stackings $k=1,4$, and $8 . k=2$ has a small relative interface energy without any substitutions, about $0.5 \mathrm{~J} / \mathrm{m}^{2}$, compared to the other stackings [26]. Additionally, $k=2$ has favorable
$\mathrm{W} \rightarrow$ Ti substitutions in $\mathrm{Ml}_{1}$. Based on this, the most likely stackings are $k=1,2,4$, and 8 .

For these four different stackings we construct CEs using ICET [21]. First, 50 initial structures are generated randomly and from these an initial cluster expansion is constructed which is used to find low-energy structures. These low-energy structures are added as training structures and this procedure is repeated a few times until convergence is reached, which amounted to about 200 training structures for each stacking. We use an interaction cutoff of 5 a nd $3.5 \AA$ for pairs and triplets, respectively. This results in about 80 parameters in total, about 50 of which correspond to pairs and 30 to triplets. The triplet interaction is generally smaller than the pair interactions but crucial to obtain accurate models. The accuracy of the models is evaluated using cross validation $(\mathrm{CV})$, and we obtain root-mean-square errors (RMSEs) of about $6 \mathrm{meV} / \mathrm{site}$, for all $k$ 's, which is equivalent to about $0.05 \mathrm{~J} / \mathrm{m}^{2}$. In the Supplemental Material [27] more details on the CEs errors are provided.

The ground-state configuration for a given $x_{\mathrm{Va}}$ and $x_{\mathrm{Ti}}$, i.e., the configuration that minimizes $\Delta \gamma_{k}^{0}\left(x_{\mathrm{Va}}, x_{\mathrm{Ti}}\right)$, is found through the mixed integer programming functionality in ICET [21]. This approach has been demonstrated to be an effective method for finding the true ground states of CEs [39]. The resulting ground-state energies $\Delta \gamma_{k}^{0}\left(x_{\mathrm{Va}}, x_{\mathrm{Ti}}\right)$ are shown in Fig. 4.

The minimum for $\Delta \gamma_{k}^{0}$ is at $x_{\mathrm{Ti}}=50 \%$ for $k=4$. An important and intriguing result is that this ground-state structure does not have mixed $(\mathrm{Ti}, \mathrm{W})$ metal layers. Instead, the structure consists of a full W topmost metal layer $\left(\mathrm{Ml}_{1}\right)$ towards Co and then, below, a full Ti metal layer $\left(\mathrm{Ml}_{2}\right)$.

Both stacking $k=2$ and 4 show negative values for $\Delta \gamma$ for Ti concentrations around $50 \%$. This indicates that at $0 \mathrm{~K}$ (and 


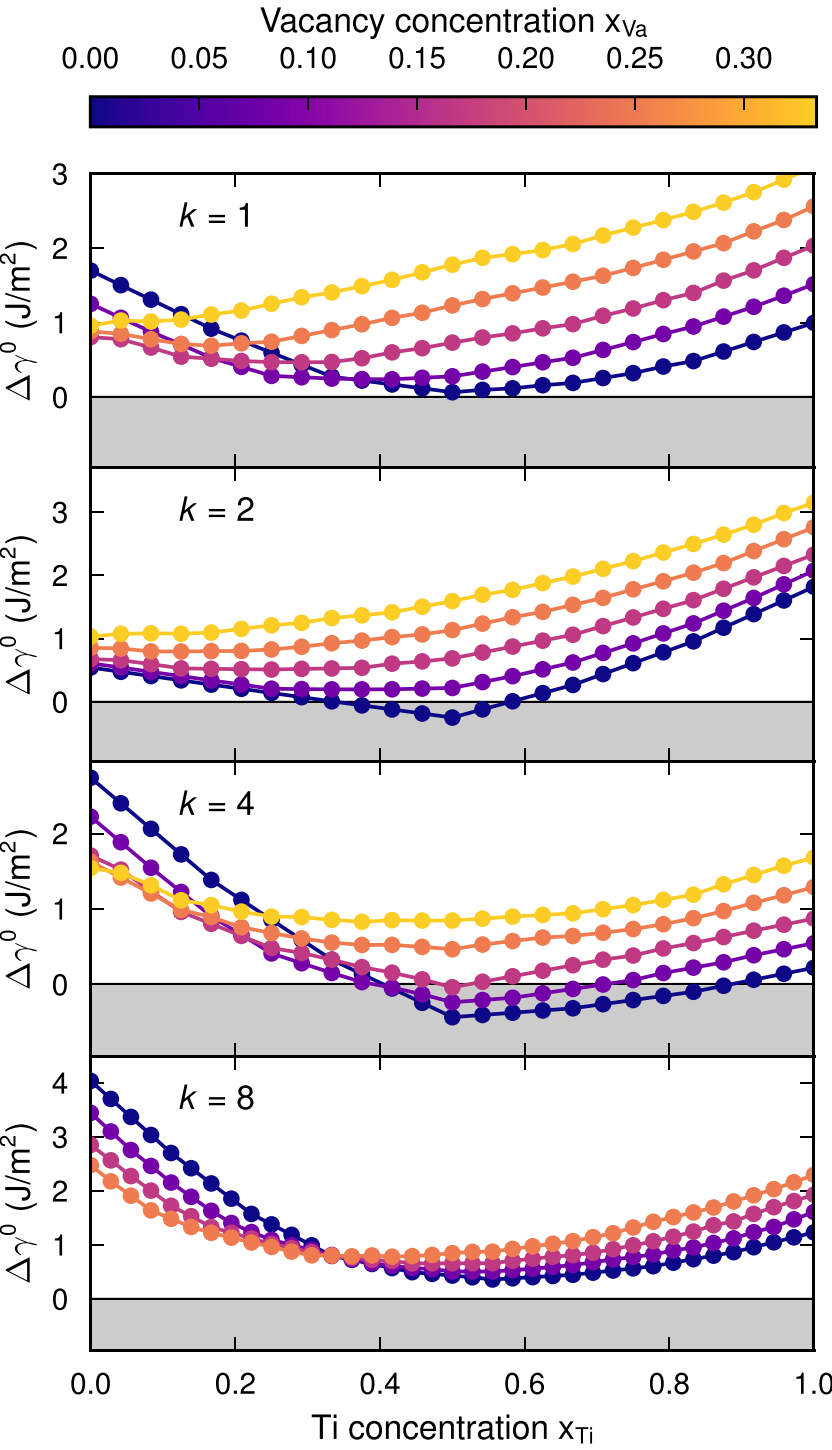

FIG. 4. Relative interface energy at $0 \mathrm{~K}, \Delta \gamma_{k}^{0}\left(x_{\mathrm{Va}}, x_{\mathrm{Ti}}\right)$, for stackings $k=1,2,4$, and 8 . The coloring indicates the carbon vacancy concentration. The Ti concentration is given relative to the number of mixing sites (see Sec. II). Shaded regions indicate where $\Delta \gamma$ becomes negative and thus where the films are energetically favorable compared to $k=0$.

with $\Delta \mu_{\mathrm{C}}=0$ and $\left.\Delta \mu_{\mathrm{Ti}}=0\right)$ a thin-film structure would be stable. For stacking $k=2$ small negative $\Delta \gamma$ values are obtained while for stacking $k=4$ considerably smaller values are obtained and also for a wider range of both $\mathrm{Ti}$ and vacancy concentrations. Based on this we only consider stacking $k=4$ in our complete analysis of the temperature dependence.

\section{B. Temperature-dependent contributions}

Next we consider the temperature-dependent contribution to the relative interface energy, the configurational part $\Delta \gamma_{k}^{\text {conf }}\left(x_{\mathrm{Va}}, x_{\mathrm{Ti}}, T\right)$ defined in Eq. (12), and the vibrational part $\Delta \gamma_{k}^{\mathrm{vib}}\left(x_{\mathrm{Va}}, x_{\mathrm{Ti}}, T\right)$ defined in Eq. (13). In Fig. 5 these free energies are shown at $1670 \mathrm{~K}$ as a function of Ti concentration and for various $\mathrm{C}$ vacancy concentrations for $k=4$.

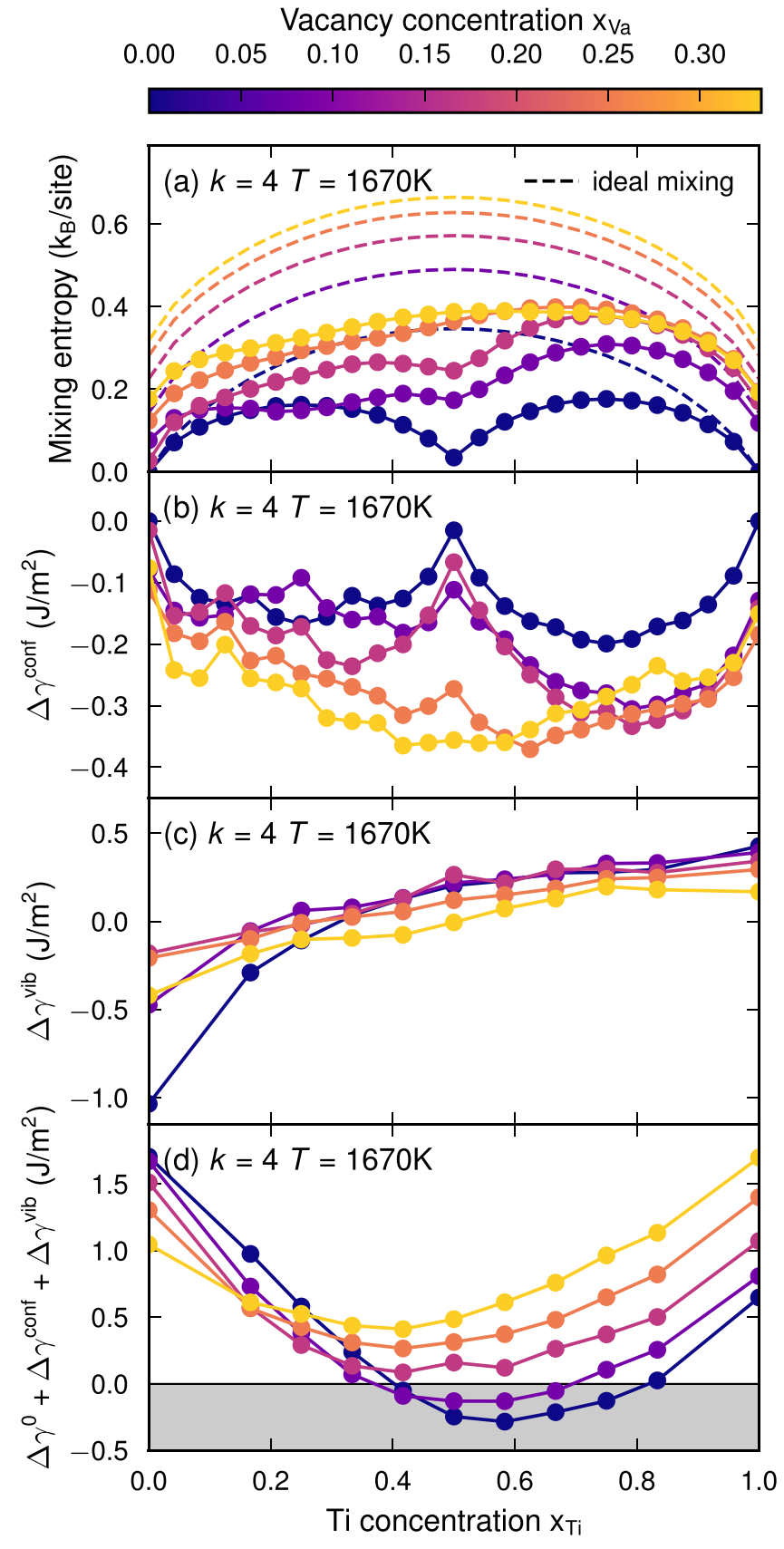

FIG. 5. Temperature-dependent contributions to the relative interface energy for $k=4$. (a) Mixing entropy at $1670 \mathrm{~K}$. (b) Configurational part to relative interface energy $\Delta \gamma^{\text {conf }}$ at $1670 \mathrm{~K}$. (c) Vibrational part to relative interface energy $\Delta \gamma^{\text {vib }}$ at $1670 \mathrm{~K}$.

(d) Relative interface energy including the $0 \mathrm{~K}$ contribution and the temperature-dependent contributions at $1670 \mathrm{~K}$.

The mixing entropy at this temperature deviates from the ideal mixing entropy and further shows significant variation with respect to $\mathrm{Ti}$ concentration. Specifically, at $x_{\mathrm{Ti}}=$ $0.5, x_{\mathrm{Va}}=0$ the entropy drops, indicating a strongly bound ground-state structure at this composition. The behavior of the mixing entropy is reflected in $\Delta \gamma^{\text {conf }}$, which approaches zero around $x_{\mathrm{Ti}}=0.5$ for $x_{\mathrm{Va}}=0$. When $\mathrm{C}$ vacancies are introduced the entropy naturally increases and more so for high Ti concentrations. 
Without $\mathrm{Ti}$ in the film the vibrational free energy $\Delta \gamma^{\text {vib }}$ arises mainly from the large vibrational free-energy difference between $\delta$-WC and $\gamma$-WC [38]. Introducing vacancies increases the vibrational free energy, also observed in cubic films in undoped cemented carbides in Ref. [26]. When Ti is introduced, more atoms in the films behave as the reference

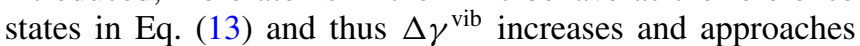
zero. The fact that $\Delta \gamma^{\text {vib }}$ becomes positive indicates that atoms in the film yield higher vibrational frequencies and thus a higher vibrational free energy than the reference states. This can partially be understood from the fact that the $\mathrm{TiC}$ film is strained (compressed) and thus slightly higher harmonic frequencies are expected, which leads to a higher vibrational free energy.

Next, the temperature-dependent parts $\Delta \gamma_{k}^{\text {vib }}$ and $\Delta \gamma_{k}^{\text {conf }}$ can be added to the $0 \mathrm{~K}$ contribution $\Delta \gamma_{k}^{0}$, and the resulting interface energy is shown in Fig. 5(d).

We restricted our detailed temperature-dependent analysis to one stacking, $k=4$. It was based on that stacking $k=4$ is more energetically favorable compared with stacking $k=2$ at $0 \mathrm{~K}$, where the interface energy difference between the two is $0.19 \mathrm{~J} / \mathrm{m}^{2}$ (Fig. 4). The vibrational free-energy contributions $\Delta \gamma^{\mathrm{vib}}$ are $0.2 \mathrm{~J} / \mathrm{m}^{2}$ for $k=4$ with $x_{\mathrm{Ti}}=0.5$ and $x_{\mathrm{Va}}=0$ at $1670 \mathrm{~K}$. A single vibrational free-energy calculation was carried out for the ground-state structure for $k=2$, with $x_{\mathrm{Ti}}=0.5$ and $x_{\mathrm{Va}}=0$, which gave $\Delta \gamma^{\mathrm{vib}}=0.29 \mathrm{~J} / \mathrm{m}^{2}$ at $1670 \mathrm{~K}$, i.e., a larger value than for $k=4$. Hence, the vibrational free energy increases the interface energy of $k=2$ relative to $k=4$. Further, like the $k=4$ ground state at $x_{\mathrm{Ti}}=$ 0.5 and $x_{\mathrm{Va}}=0$ the corresponding ground state for $k=2$ is also strongly bound, meaning a low configurational entropy and a small effect from configurational free energy. Therefore, the $k=2$ stacking would most likely not become favorable including temperature effects, and the choice to restrict the full temperature-dependent analysis to $k=4$ is well motivated.

\section{Environmental-dependent contribution}

Finally, we consider the environmental contribution to the relative interface energy $\Delta \gamma_{k}^{\text {env }}\left(x_{\mathrm{Va}}, x_{\mathrm{Ti}}\right)$, defined in Eq. (14). By adding this to the other parts we obtain the total relative interface energy $\Delta \gamma_{k}\left(x_{\mathrm{Va}}, x_{\mathrm{Ti}}, T\right)$, which then depends parametrically on $\Delta \mu_{\mathrm{C}}$ and $\Delta \mu_{\mathrm{Ti}}$. The contribution from $\Delta \gamma_{k}^{\text {env }}$ and the resulting $\Delta \gamma_{k}$ is shown in Fig. 6 for $k=4$ using $\Delta \mu_{\mathrm{C}}=-0.15 \mathrm{eV}$ and $\Delta \mu_{\mathrm{Ti}}=0.05 \mathrm{eV}$ at $T=1670 \mathrm{~K}$. We notice that the thin film becomes stable at $1670 \mathrm{~K}$ with a Ti concentration $x_{\mathrm{Ti}}$ slightly larger than 0.5 .

The last step is to obtain the relative interface energy as a function of $\Delta \mu_{\mathrm{C}}$ and $\Delta \mu_{\mathrm{Ti}}, \Delta \gamma_{k}\left(\Delta \mu_{\mathrm{C}}, \Delta \mu_{\mathrm{Ti}}, T\right)$. Following Eq. (15) we therefore minimize $\Delta \gamma_{k}\left(x_{\mathrm{Va}}, x_{\mathrm{Ti}}, T\right)$ with respect to both $x_{\mathrm{Va}}$ and $x_{\mathrm{Ti}}$, given $\Delta \mu_{\mathrm{C}}$ and $\Delta \mu_{\mathrm{Ti}}$. The minimization yields concentrations in the range $x_{\mathrm{Va}}=0-0.05$ and $x_{\mathrm{Ti}}=0.42-0.58$. In Fig. 7 the result for $\Delta \gamma\left(\Delta \mu_{\mathrm{C}}, \Delta \mu_{\mathrm{Ti}}, T\right)$ for stacking $k=4$ is shown at $T=1670 \mathrm{~K}$. It is clear that $\Delta \gamma\left(\Delta \mu_{\mathrm{C}}, \Delta \mu_{\mathrm{Ti}}, T\right)$ mainly depends on the sum $\Delta \mu_{\mathrm{Ti}}+$ $\Delta \mu_{\mathrm{C}}$. This is expected as the concentrations $x_{\mathrm{Va}}$ and $x_{\mathrm{Ti}}$ in the film do not vary much and thus $\Delta \gamma$ have an almost linear dependency on the chemical potentials [see Eq. (14)]. This results in a thermodynamic stability region for the film $\Delta \gamma<0$, for which $\Delta \mu_{\mathrm{Ti}}+\Delta \mu_{\mathrm{C}}$ is almost constant at approximately $-0.12 \mathrm{eV} /$ atom . The stability limit of the thin film is shown
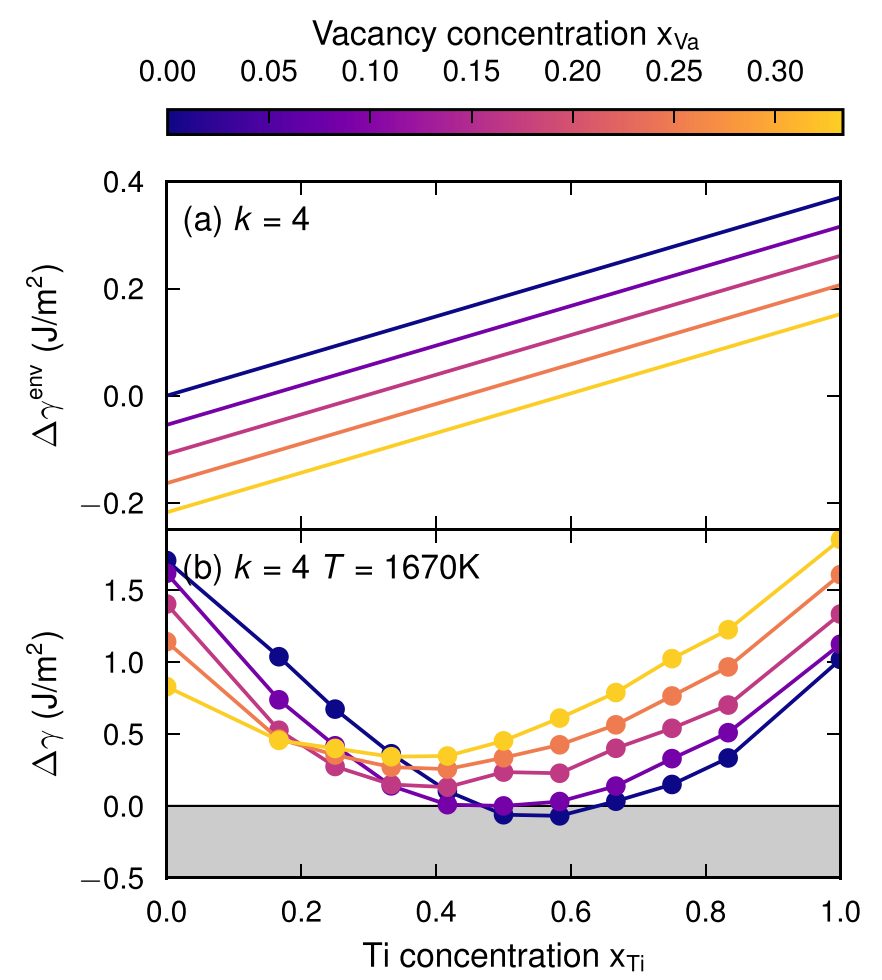

FIG. 6. Relative interface energy $\Delta \gamma\left(x_{\mathrm{Va}}, x_{\mathrm{Ti}}, T\right)$ at $T=1670 \mathrm{~K}$ for $k=4$. (a) The contribution from $\Delta \gamma^{\text {env }}$ using $\Delta \mu_{\mathrm{C}}=-0.15 \mathrm{eV}$ and $\Delta \mu_{\mathrm{Ti}}=0.05 \mathrm{eV}$. (b) Relative interface energy $\Delta \gamma\left(x_{\mathrm{Va}}, x_{\mathrm{Ti}}, T\right)$, using $\Delta \mu_{\mathrm{C}}=-0.15 \mathrm{eV}$ and $\Delta \mu_{\mathrm{Ti}}=0.05 \mathrm{eV}$.

by the red line in Fig. 7 and the red dashed line shows the stability limit neglecting the vibrational contribution $\Delta \gamma^{\text {vib }}$. This shows that the vibrational free energy has a rather large impact on the stability region for the cubic films.

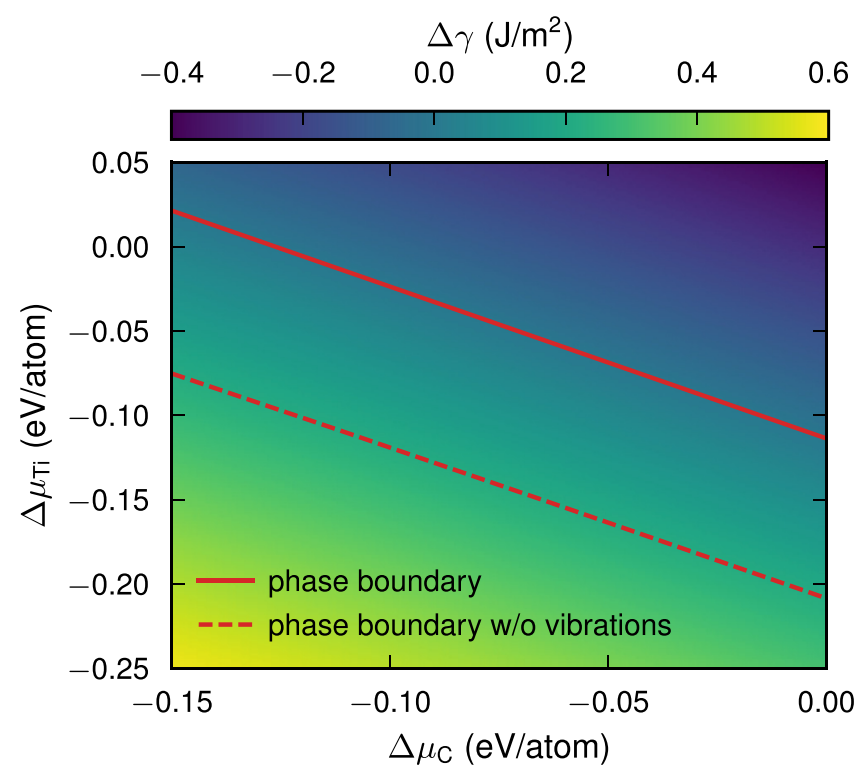

FIG. 7. Relative interface energy $\Delta \gamma\left(\Delta \mu_{\mathrm{C}}, \Delta \mu_{\mathrm{Ti}}, T\right)$ at $T=$ $1670 \mathrm{~K}$ for $k=4$. The red line indicate the phase boundary where $\Delta \gamma$ goes from negative to positive. The red dashed line indicates this boundary if vibrations would not have been included, i.e., neglecting $\Delta \gamma^{\mathrm{vib}}$. 

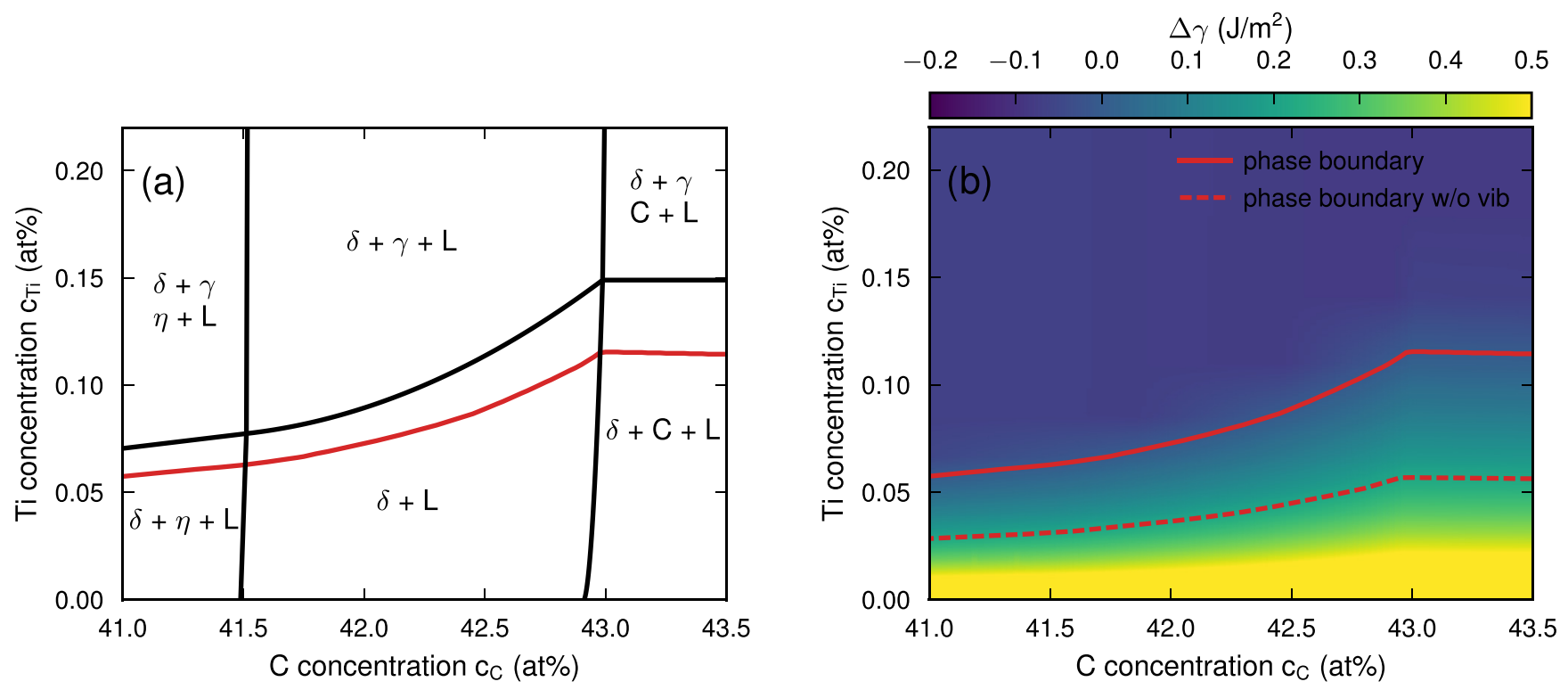

FIG. 8. To the left, the phase diagram of the present W-C-Co-Ti system generated by THERMO-CALC at $1670 \mathrm{~K}$. Here, $\delta$ refers to the hexagonal $\delta$-WC, $\gamma$ refers to the cubic $\gamma-(\mathrm{Ti}, \mathrm{W}) \mathrm{C}_{x}$ phase, $\mathrm{C}$ refers to graphite, $\mathrm{L}$ refers to the Co-rich liquid, and $\eta$ refers to eta phase. To the right, heat map of the relative interface energy $\Delta \gamma$ of the $k=4$ film at $1670 \mathrm{~K}$. The red line (in both figures) indicates the lower limit with respect to Ti concentration where cubic thin films become thermodynamically stable at the WC/Co phase boundary. The red dashed line indicates the same boundary but without vibrations included, i.e., neglecting $\Delta \gamma^{\text {vib }}$.

\section{Interfacial phase diagram}

The interfacial phase diagram shows for which compositions of a system the thin films are stable as function of temperature. To obtain that diagram we first determine the ordinary bulk phase diagram. For this we use THERMO-CALC (see Appendix B) and in Fig. 8(a) we show the result at $1670 \mathrm{~K}$ for our chosen W-C-Co-Ti composition (see Appendix B) as function of carbon $\left(c_{\mathrm{C}}\right)$ and titanium $\left(c_{\mathrm{Ti}}\right)$ atomic concentrations.

For all compositions the hexagonal WC phase $(\delta)$ and a Co-rich liquid phase (L) are stable. Graphite (C) precipitates for high carbon concentration $\left(c_{\mathrm{C}} \gtrsim 43.0\right.$ at.\%) and for low carbon concentration $\left(c_{\mathrm{C}} \lesssim 41.5\right.$ at.\%) the eta phase $(\eta)$ forms. The region in-between these two limits is the so-called "carbon window." When increasing the Ti concentration the $\gamma-(\mathrm{Ti}, \mathrm{W}) \mathrm{C}_{x}$ precipitates. In the graphite limit it starts to precipitate at $c_{\mathrm{Ti}}=0.15$ at. $\%$ with composition $\left(\mathrm{Ti}_{0.65} \mathrm{~W}_{0.35}\right) \mathrm{C}_{0.93}$ and in the eta limit at $c_{\mathrm{Ti}}=0.077$ at. $\%$ with composition $\left(\mathrm{Ti}_{0.61} \mathrm{~W}_{0.39}\right) \mathrm{C}_{0.90}$.

The next step is to add the information about the film stability and create the interfacial phase diagram. We transform the relative interface energy to a function of atomic concentrations

$$
\Delta \gamma_{k}\left(\Delta \mu_{\mathrm{C}}, \Delta \mu_{\mathrm{Ti}}, T\right) \rightarrow \Delta \gamma_{k}\left(c_{\mathrm{C}}, c_{\mathrm{Ti}}, T\right),
$$

using the data presented in Fig. 3. The result for $\Delta \gamma_{k}\left(c_{\mathrm{C}}, c_{\mathrm{Ti}}, T\right)$ is added to the bulk phase diagram in Fig. 8(a). The red line shows the stability limit for the thin-film structure with stacking $k=4$. For higher Ti concentrations the $k=4$ film becomes thermodynamically stable $(\Delta \gamma<0)$ and for lower Ti concentrations it becomes thermodynamically unstable $(\Delta \gamma>0)$. In the graphite limit the film becomes stable for Ti concentrations larger than $c_{\mathrm{Ti}}=0.115$ at. $\%$ and in the eta limit the corresponding concentration is $c_{\mathrm{Ti}}=0.062$ at. $\%$. It is important to note that the thin-film structure becomes stable for lower Ti concentrations than required for the bulk phase $\gamma-(\mathrm{Ti}, \mathrm{W}) \mathrm{C}_{x}$ to precipitate.

In Fig. 8(b) we show $\Delta \gamma\left(c_{\mathrm{C}}, c_{\mathrm{Ti}}, T\right)$ as a heat map at $1670 \mathrm{~K}$. As in Fig. 8(a) the red line shows the stability limit $(\Delta \gamma=0)$ and the red dashed line indicates the same boundary neglecting the vibrational contribution. We note that the vibrational effect has a significant impact. The critical concentration $c_{\mathrm{Ti}}$ for which films become stable increases with almost a factor of 2 when including vibrations.

Additionally, the same analysis is carried out for $1300 \mathrm{~K}$ for which $\mathrm{Co}$ is in a solid face-centered-cubic (fcc) phase. The resulting phase diagram is shown in Fig. 9. Note that the scale for the Ti concentration is about one order less compared to the phase diagram at $1670 \mathrm{~K}$ in Fig. 8. Here, the carbon window is a bit narrower compared to $1670 \mathrm{~K}$. The Ti concentrations for which $\gamma$ phase is stable is $c_{\mathrm{Ti}}=0.0037$ at. $\%$ and $c_{\mathrm{Ti}}=0.013$ at. $\%$ in the graphite and eta limit, respectively. In the graphite limit the film becomes stable for $\mathrm{Ti}$ concentrations larger than $c_{\mathrm{Ti}}=0.0011$ at.\% and in the eta limit the corresponding concentration is $c_{\mathrm{Ti}}=0.0039$ at. $\%$. Hence, the film is stable for Ti concentrations below the solubility limit in the binder phase also at solid-state sintering temperatures.

For both 1300 a nd $1670 \mathrm{~K}$ the relative interface energy $\Delta \gamma$ decreases gradually when the $\mathrm{Ti}$ concentration $c_{\mathrm{Ti}}$ increases from zero. This is due to the fact that the chemical potential of Ti, $\Delta \mu_{\mathrm{Ti}}$, increases with increasing Ti concentration (see Fig. 3) which yields a reduction in $\Delta \gamma$ according to Eq. (14). Once $\gamma$-(Ti,W) $C_{x}$ becomes stable, the chemical potential of $\mathrm{Ti}$ becomes essentially constant even when increasing the Ti concentration. Consequently, the relative interface energy $\Delta \gamma$ also becomes constant for all $\mathrm{Ti}$ concentrations above the stability limit for $\gamma-(\mathrm{Ti}, \mathrm{W}) \mathrm{C}_{x}$. See Fig. S5 in the Supplemental 

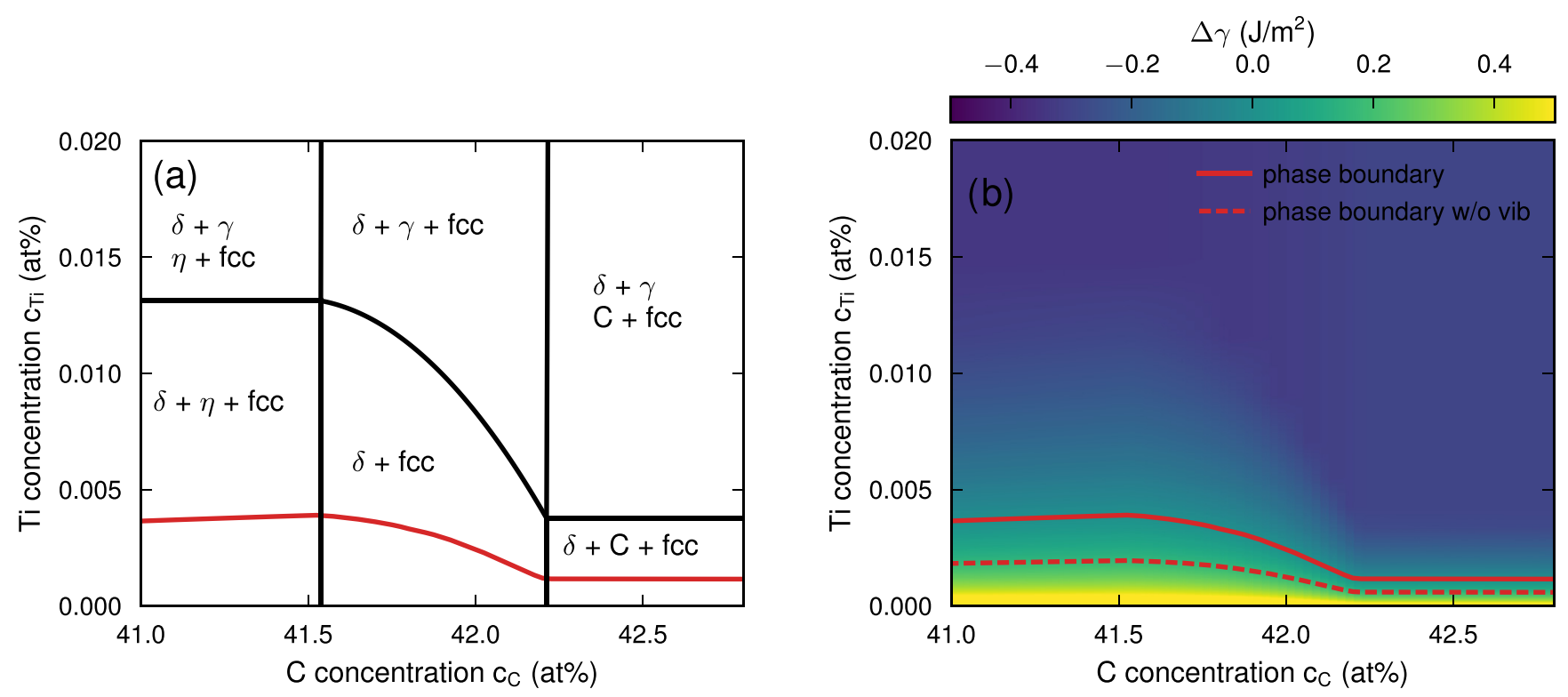

FIG. 9. To the left, the phase diagram of the present W-C-Co-Ti system generated by THERMO-CALC at $1300 \mathrm{~K}$. Here fcc refers to the solid Co fcc phase. To the right, heat map of the relative interface energy $\Delta \gamma$ of the $k=4$ film at $1300 \mathrm{~K}$. The red line (in both figures) indicates the lower limit with respect to Ti concentration where cubic thin films become thermodynamically stable at the WC/Co phase boundary. The red dashed line indicates the same boundary but without vibrations included, i.e., neglecting $\Delta \gamma^{\text {vib }}$.

Material [27] for more exactly how $\Delta \gamma$ and $\Delta \mu_{\mathrm{Ti}}$ vary as a function of Ti concentration $c_{\mathrm{Ti}}$.

\section{E. Ordering of films}

We found in Sec. V A that at $0 \mathrm{~K}$ for stacking $k=4$ the Ti atoms exclusively occupied the second metal layer and no $\mathrm{Ti}$ atoms were found to occupy the first metal layer. Here, we will now explore the temperature dependence of the occupation of the two sublattices $(\mathrm{W}, \mathrm{Ti})$ and $(\mathrm{C}, \mathrm{Va})$. This is done by carrying out $\mathrm{MC}$ simulations in the semi-grand-canonical ensemble as a function of temperature. We use $\Delta \mu_{\mathrm{C}}=-0.15 \mathrm{eV}$ and $\Delta \mu_{\mathrm{Ti}}=0.05 \mathrm{eV}$, which are representative values for a system close to the formation of the eta phase. The result is seen in Fig. 10 (left).

When increasing the temperature some Ti atoms are mixed into the topmost metal layer $\left(\mathrm{Ml}_{1}\right)$ while the second metal layer $\left(\mathrm{Ml}_{2}\right)$ is essentially occupied by only $\mathrm{Ti}$ atoms. At $1670 \mathrm{~K} \mathrm{Ml}_{1}$ is occupied by $90 \% \mathrm{~W}$ atoms and $\mathrm{Ml}_{2}$ by $99 \%$ $\mathrm{Ti}$ atoms. Some carbon vacancies are introduced, about $2 \%$ in $\mathrm{Cl}_{1}$ and less than $0.5 \%$ in $\mathrm{Cl}_{2}$, at $1670 \mathrm{~K}$. In Fig. 10 (right) a snapshot from the MC simulation at $1670 \mathrm{~K}$ is shown. The occupation changes slightly with $\Delta \mu$ and would be somewhat affected if the effect from vibrations had been included. However, the overall qualitative behavior would stay the same.

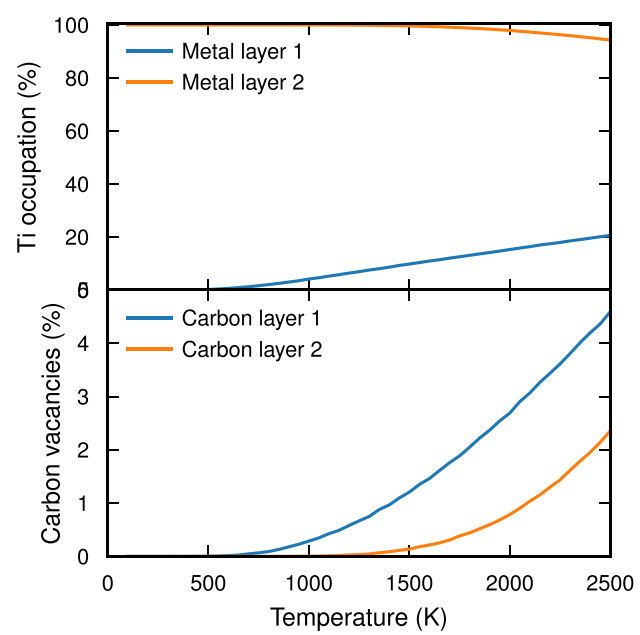

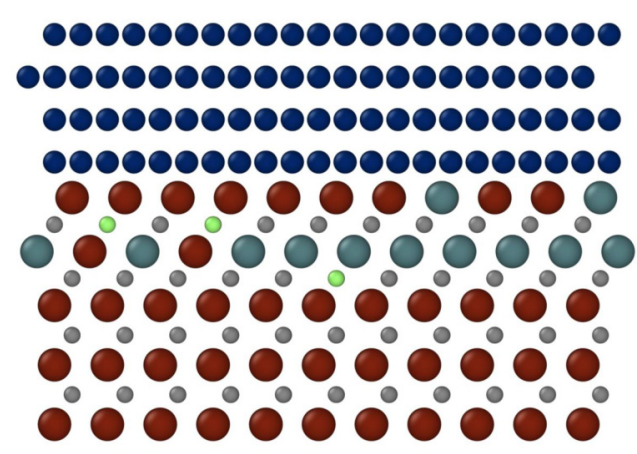

Co

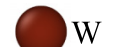

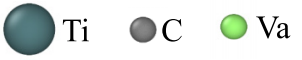

FIG. 10. To the left, the occupation of the two metal layers in stacking $k=4$ by Ti atoms (top). The occupation of the two carbon layers in stacking $k=4$ by vacancies (bottom). Both are shown as a function of temperature obtained from semi-grand canonical (SGC) MC simulations with $\Delta \mu_{\mathrm{C}}=-0.15 \mathrm{eV}$ and $\Delta \mu_{\mathrm{Ti}}=0.05 \mathrm{eV}$. To the right a high-temperature structure from the MC simulation at $1670 \mathrm{~K}$ is shown; here Va denotes carbon vacancies. 
TABLE I. Spacing between metal layers (in $\AA$ ) compared with experimental data. The theoretical calculations are done for two types of structures: the ground-state structure, i.e., a low-temperature structure (LT) and a high-temperature structure (HT) generated from a MC simulation at $1670 \mathrm{~K}$. In both cases the thin film has the $k=4$ stacking.

\begin{tabular}{lcccc}
\hline \hline & \multicolumn{2}{c}{ Theory } & \multicolumn{2}{c}{ Experiment } \\
& LT & HT & Ref. [18] & Ref. [19] \\
& 2.60 & 2.57 & 2.47 & 2.6 \\
$\mathrm{Ml}_{1}-\mathrm{Ml}_{2}$ & 2.63 & 2.63 & 2.64 & 2.6 \\
$\mathrm{Ml}_{2}-\mathrm{Ml}_{3}$ & 2.85 & 2.85 & 2.82 & 3.0 \\
$\mathrm{Ml}_{3}-\mathrm{Ml}_{4}$ & 2.85 & 2.85 & 2.82 & 3.0 \\
$\mathrm{Ml}_{4}-\mathrm{Ml}_{5}$ & & \\
\hline \hline
\end{tabular}

We have also calculated the spacing between the metal layers for two types of structures, a low-temperature (LT) and a high-temperature (HT) structure. The LT structure corresponds the ground-state structure, meaning the lowest-energy structure at $0 \mathrm{~K}$. It contains no carbon vacancies and has $100 \% \mathrm{~W}$ atoms in the first metal layer $\mathrm{Ml}_{1}$ and $100 \% \mathrm{Ti}$ atoms in the second metal layer $\mathrm{Ml}_{2}$. The $\mathrm{HT}$ structure is extracted from the MC simulation in the SGC ensemble used for Fig. 10 at $1670 \mathrm{~K}$. It contains a small amount of carbon vacancies and $\mathrm{W}$ and $\mathrm{Ti}$ atoms mix to some extent in the metal layers. The layer spacing for the LT and HT structures is calculated by relaxing the structures using DFT. The resulting layer spacings are presented in Table I.

The spacings between layers $\mathrm{Ml}_{1}$ and $\mathrm{Ml}_{2}$ and between layers $\mathrm{Ml}_{2}$ and $\mathrm{Ml}_{3}$ are significantly smaller compared with the $\delta$-WC bulk spacing (2.85 $\AA$ ) which is found between $\mathrm{Ml}_{3}$ and $\mathrm{Ml}_{4}$ and between $\mathrm{Ml}_{4}$ and $\mathrm{Ml}_{5}$. The spacing between $\mathrm{Ml}_{1}$ and $\mathrm{Ml}_{2}$ and between $\mathrm{Ml}_{2}$ and $\mathrm{Ml}_{3}$, which have a cubic stacking (see Fig. 1), lies between the bulk spacing for stoichiometric TiC, $2.58 \AA$, and stoichiometric $\gamma$-WC, $2.75 \AA$. Here, the bulk spacings are calculated including a Poisson effect due to the strain to match with $\delta$-WC. The difference in spacing between LT and HT is small and comes from the fact that the composition and ordering of the film at $1670 \mathrm{~K}$ deviates slightly from the $0 \mathrm{~K}$ structure (see Fig. 10).

In Table I we also compare our theoretical data with the experimental results from Refs. [18,19]. In experiments a material sintered at liquid-phase sintering temperatures is studied at room temperature. If one assumes that the composition and ordering do not change in the cooling process our HT results should reflect the experimental situation, otherwise, it should be more close to the LT results. Our results are in good qualitative agreement with the experimental studies which both find that the two topmost layer spacings are reduced in size compared with the bulk value.

\section{DISCUSSION}

\section{A. Structure and composition of the thin film}

The thin films are stabilized by interfacial effects. In Ref. [40] a simplified model was introduced to illustrate this idea. Consider a phase boundary between hexagonal WC $(\delta$ WC) and Co, i.e., a phase boundary without any thin-film structure. Denote the interface energy as $\gamma_{\text {no film }}=\gamma_{\delta \text {-WC/Co }}$.
For a phase boundary with a thin film consisting of $N$ layers of stoichiometric $\mathrm{TiC}$, the interface energy can be approximated as

$$
\gamma_{\text {film }}=\gamma_{\delta-\mathrm{WC} / \mathrm{TiC}}+N(\Delta g+e)+\gamma_{\mathrm{TiC} / \mathrm{Co}},
$$

where $\Delta g$ is the free-energy cost to create bulk TiC per layer of $\mathrm{TiC}$ (one metal layer and one carbon layer) and $e$ is the corresponding elastic energy associated with the formation of the thin film. The relative interface energy $\Delta \gamma$, the cost to create a thin film, can then be written as

$$
\Delta \gamma \equiv \gamma_{\text {film }}-\gamma_{\text {no film }}=\Gamma+N(\Delta g+e)
$$

where

$$
\Gamma=\gamma_{\delta-\mathrm{WC} / \mathrm{TiC}}+\gamma_{\mathrm{TiC} / \mathrm{Co}}-\gamma_{\delta-\mathrm{WC} / \mathrm{Co}} .
$$

If $\Delta \gamma$ is negative, the interface effects outweigh the bulk cost and film formation is favorable. This simplified model gave a semiquantitative description for $N \geqslant 2$ (cf. Ref. [40]).

Let us first consider the value of $\Gamma$. Interface energies have been calculated or collected from a previous study [26] and are presented in Table 1 in the Supplemental Material [27]. Using these values we obtain $\Gamma=-0.61 \mathrm{~J} / \mathrm{m}^{2}$. This negative value of $\Gamma$ implies a tendency for film formation. However, we have to add the cost to create the $\mathrm{TiC}$ layers. We assume equilibrium with bulk TiC, i.e., $\Delta g=0$, and the corresponding strain energy is $e=0.40 \mathrm{~J} / \mathrm{m}^{2}$ per TiC layer (see Supplemental Material [27]). Assuming $N=2$ we obtain a rather low relative interface energy $\Delta \gamma=0.19 \mathrm{~J} / \mathrm{m}^{2}$, but the film is not stable.

However, the interface energy for the $\mathrm{TiC} / \mathrm{Co}$ phase boundary is quite large, $\gamma_{\mathrm{TiC} / \mathrm{Co}}=0.86 \mathrm{~J} / \mathrm{m}^{2}$, compared to phase boundaries between some other cubic carbides and cobalt (see Ref. [41]). In particular, the phase boundary between cubic WC $(\gamma-\mathrm{WC})$ and Co is considerably lower, $\gamma_{\gamma-\mathrm{WC} / \mathrm{Co}}=$ $-1.30 \mathrm{~J} / \mathrm{m}^{2}$ (Ref. [26]). Consider now instead the formation of a mixed $(\mathrm{Ti}, \mathrm{W}) \mathrm{C}_{x}$ thin film where $\mathrm{W}$ occupies most sites in the first metal layer $\left(\mathrm{Ml}_{1}\right)$. A phase boundary towards Co with low energy is then obtained, which may stabilize a thin-film structure even though there is a substantial cost in creating cubic WC [26]. Furthermore, the phase boundary energy between hexagonal and cubic WC is quite high [26], $\gamma_{\delta \text {-WC } / \gamma \text {-WC }}=0.45 \mathrm{~J} / \mathrm{m}^{2}$, while the corresponding energy between hexagonal $\mathrm{WC}$ and TiC is low, $\gamma_{\delta \text {-WC/TiC }}=-0.34 \mathrm{~J} / \mathrm{m}^{2}$ . This suggests having mostly $\mathrm{Ti}$ in the second metal layer $\left(\mathrm{Ml}_{2}\right)$ in the mixed film.

Indeed, this is exactly what happens in our detailed modeling, where $\mathrm{Ti}$ and $\mathrm{W}$ atoms are allowed to mix freely within the thin-film structure. The equilibrium $(\mathrm{Ti}, \mathrm{W}) \mathrm{C}_{x}$ thin film has a thickness of two cubic layers with essentially only $\mathrm{W}$ in the first metal layer $\left(\mathrm{Ml}_{1}\right)$ and virtually only $\mathrm{Ti}$ in the second metal layer $\left(\mathrm{Ml}_{2}\right)$ (see Fig. 10).

Adding an additional $\mathrm{TiC}$ layer $\left(\right.$ at $\mathrm{Ml}_{3}$ and $\mathrm{Cl}_{3}$ ) would cost about $0.4 \mathrm{~J} / \mathrm{m}^{2}$, corresponding to the strain energy needed to compress $\mathrm{TiC}$ into coincidence with the hexagonal $\mathrm{WC}$ lattice, and thus is unlikely to happen. Moreover, adding an extra cubic WC layer is also very unlikely due to its high bulk energy compared to hexagonal WC [26]. Thinner films are also unlikely since at least two cubic layers are needed 
in order to optimize interface energies compared to the bulk cost. Removing either the W-rich layer toward Co $\left(\mathrm{Ml}_{1}\right)$ or the Ti-rich layer $\left(\mathrm{Ml}_{2}\right)$ increases $\Delta \gamma$.

\section{B. Stability of the thin film}

We have determined the thermodynamic stability of thin cubic films at the basal plane for two different temperatures, 1300 a nd $1670 \mathrm{~K}$. These two temperatures correspond to solid-state and liquid-state sintering conditions, respectively. The film is predicted to be stable for Ti concentrations larger than a critical value $c_{\mathrm{Ti}}^{\text {crit }}$. At both temperatures this critical value $c_{\mathrm{Ti}}^{\text {crit }}$ is lower than the Ti concentration that is required for the corresponding bulk $\gamma-(\mathrm{Ti}, \mathrm{W}) \mathrm{C}_{x}$ to precipitate. This is important, and shows that the thin films can be present at the basal plane both at solid-state and liquid-state sintering conditions.

Consider now the system at $1670 \mathrm{~K}$ and the interfacial phase diagram in Fig. 8. Assume the carbon concentration 42.0 at. $\%$. By increasing the Ti concentration from zero the relative interface energy $\Delta \gamma$ gradually decreases. At the Ti concentration $c_{\mathrm{Ti}}^{\text {crit }}=0.072$ at. $\%$ it becomes zero, $\Delta \gamma=0$, and the thin film becomes thermodynamically stable. This Ti concentration is below the solubility limit for the bulk $\gamma-(\mathrm{Ti}, \mathrm{W}) \mathrm{C}_{x}$ phase. By increasing the Ti concentration further the $\gamma$-(Ti, W $\mathrm{C}_{x}$ phase starts to precipitate at the Ti concentration $c_{\mathrm{Ti}}=0.090$ at. $\%$, the solubility limit, and at that $\mathrm{Ti}$ concentration the relative interface energy has decreased to $\Delta \gamma=-0.08 \mathrm{~J} / \mathrm{m}^{2}$. When increasing the Ti concentration further, beyond the solubility limit, the $\gamma-(\mathrm{Ti}, \mathrm{W}) \mathrm{C}_{x}$ phase continues to precipitate and the thin-film structure will be kept unchanged with $\Delta \gamma=-0.08 \mathrm{~J} / \mathrm{m}^{2}$.

At $1300 \mathrm{~K}$ the solubility limit of the bulk $\gamma-(\mathrm{Ti}, \mathrm{W}) \mathrm{C}_{x}$ phase is significantly decreased, with about a factor of 10 (see Fig. 9). The critical value for film formation $c_{\mathrm{Ti}}^{\text {crit }}$ is also decreased and also at $1300 \mathrm{~K}$ the thin-film structure becomes stable for lower $\mathrm{Ti}$ concentrations than required for the bulk $\gamma-(\mathrm{Ti}, \mathrm{W}) \mathrm{C}_{x}$ to precipitate. For the carbon concentration 42.0 at. \% we obtain the critical concentration $c_{\mathrm{Ti}}^{\text {crit }}=0.0024$ at. $\%$ and the solubility limit is located at the $\mathrm{Ti}$ concentration $c_{\mathrm{Ti}}=0.0082$ at. $\%$. At the solubility limit the relative interface energy has decreased to about $\Delta \gamma=-0.35 \mathrm{~J} / \mathrm{m}^{2}$.

The thin films are stabilized by interfacial effects. In this study the temperature dependencies have been evaluated and included in the modeling. The configurational part $\Delta \gamma^{\text {conf }}$ is found to make only a small contribution, while the vibrational

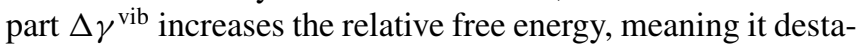
bilizes the thin-film structure. This is seen in Figs. 8 and 9 where the red dashed lines show the stability boundary for thin-film formation without vibrations included.

The first metal layer $\left(\mathrm{Ml}_{1}\right)$ contains some $\mathrm{Ti}$ atoms but predominantly $\mathrm{W}$ atoms, while the second metal layer $\left(\mathrm{Ml}_{2}\right)$ contains essentially only $\mathrm{Ti}$ atoms (see Fig. 10). The carbon layers $\left(\mathrm{Cl}_{1}\right.$ and $\left.\mathrm{Cl}_{2}\right)$ contain only a small amount of carbon vacancies. This is in contrast to WC-Co without dopants, where cubic WC ( $\gamma$-WC) thin films with up to $50 \%$ carbon vacancies are obtained at liquid-phase sintering temperatures at low carbon content (eta limit). At high carbon content (graphite limit), the cubic WC $(\gamma-\mathrm{WC})$ thin films are absent [26].

\section{Comparison with experiments}

Several high-resolution microscopy studies of Ti-doped WC-Co have recently been performed, which give atomicscale information on the interfaces in the material $[12,18,19]$. Meingast et al. [18] studied a material with Ti concentration $c_{\mathrm{Ti}}=0.35$ at. \%, Yang et al. [19] with 0.38 at. $\%$ on metal basis (i.e., $c_{\mathrm{Ti}} \simeq 0.22$ at.\% ), and Liu et al. [12] studied a material with $1.0 \mathrm{wt} . \% \mathrm{TiC}$. In all of the experimentally studied materials thin cubic films were found to be present at the phase boundary between the hexagonal WC basal plane and Co.

Our prediction is that a thin cubic film, a complexion, is present both at solid-state $(1300 \mathrm{~K})$ and liquid-phase $(1670 \mathrm{~K})$ sintering conditions at the basal plane for the Ti concentrations used in Refs. [12,18,19].

We find that the topmost metal layer in the thin film $\left(\mathrm{Ml}_{1}\right)$ contains only about $12 \% \mathrm{Ti}$ atoms at $1670 \mathrm{~K}$, and less at lower temperatures. The rest are $\mathrm{W}$ atoms. On the other hand, the second metal layer $\left(\mathrm{Ml}_{2}\right)$ consists of essentially only $\mathrm{Ti}$ atoms. The third, fourth, etc., metal layers have hexagonal stacking and contain only $\mathrm{W}$ atoms. This agrees quite well with the experimental studies by Meingast et al. [18] and Yang et al. [19], who both find that the topmost layer $\left(\mathrm{Ml}_{1}\right)$ is mainly occupied by $\mathrm{W}$ atoms and the second layer $\left(\mathrm{Ml}_{2}\right)$ by Ti atoms The experimental results in Ref. [19] indicate that the composition in these two metal layers is more mixed compared with our theoretical prediction. Also, the spacing between the metal layers is found to compare well between our theoretical data and the experimental results $[18,19]$ (see Table I).

The details of the stacking are identified by Yang et al. [19] and by Meingast et al. [18]. They both observed metal layer positions that are consistent with the $k=4$ stacking, the stacking that we predict to be the stable one. The work by Liu et al. [12] seems to indicate a noncoherent thin film and possibly also thicker films [see their Fig. 5(b)]. Although the indicated lattice spacing in their film [indicated by green dots in Fig. 5(b)] seems to be too large to be consistent with $\gamma$ - $(\mathrm{Ti}, \mathrm{W}) \mathrm{C}_{x}$.

Both Meingast et al. [18] and Yang et al. [19] detect no Ti-containing thin films at the WC prismatic planes. This is in agreement with the theoretical prediction in Ref. [42], where it was concluded that thin-film formation is only favorable at the basal plane, not at the prismatic planes.

\section{Modeling limitations}

It is important to note that our modeling is based on calculating the relative interface energy $\Delta \gamma=\gamma_{\text {film }}-\gamma_{\text {no film }}$, which is the difference in interface energy between two similar systems. Therefore, we expect some cancellation of errors in calculating $\Delta \gamma$, if corresponding errors are present in both $\gamma_{\text {film }}$ and $\gamma_{\text {no film }}$.

In all atomic-scale calculations we have a solid Co phase towards the carbide, although we present results also for temperatures at which Co is in the liquid state. However, since the geometry of the first two atomic layers towards Co is the same for the no-film stacking $(k=0)$ and all film stackings $(k=1, \ldots, 8)$, it is likely that the relative interface energy $\Delta \gamma$ is insensitive to the physical state of the binder.

Cobolt is magnetic but all of our DFT calculations are non-spin-polarized and, hence, magnetic effects are omitted. 
For the temperatures studied in this work Co is paramagnetic [43]. However, the relative interface energy $\Delta \gamma$ is found to be insensitive with respect to the magnetic state of Co. In a few test calculations we found a difference in $\Delta \gamma$ of only $0.01 \mathrm{~J} / \mathrm{m}^{2}$ or less between spin-polarized (ferromagnetic) and non-spin-polarized calculations. Therefore, we have restricted our DFT calculations to the non-spin-polarized case.

The vibrational contribution to the relative interface energy $\Delta \gamma^{\mathrm{vib}}$ is in this study treated in the harmonic approximation and thus neglecting anharmonic effects. We find that $\Delta \gamma^{\text {vib }}$ is on the order of $0.2 \mathrm{~J} / \mathrm{m}^{2}$ at $1670 \mathrm{~K}$ for the $k=4$ structure. Anharmonic contributions to the free energy are usually only important close to the melting temperature. In the present case, the melting point is high, above $3000 \mathrm{~K}$, for both $\mathrm{TiC}$ and WC. Therefore, we expect the anharmonic contributions to the relative interface energy to be quite small. Thermal expansion is not included in our modeling based on DFT. We expect this effect to also cancel out to large extent. Additionally, including thermal expansion for interface systems is a nontrivial task due to relaxation of layers.

The cubic films studied in this work are compressed in order to form a coherent interface with the hexagonal WC lattice, which gives rise to elastic energy. It is therefore possible that relieving the strain by creating an incoherent interface between $\delta$-WC and the cubic film could yield a lower relative interface energy $\Delta \gamma$ and thus further stabilize the cubic films. Although, it should be noted that cubic films observed experimentally by Yang et al. [19] are coherent [see their Figs 1(c), S5, and S6].

\section{CONCLUSION}

In this paper, we compute, using ab initio calculations and modeling, interface free energies for thin cubic films in Ti-doped WC-Co cemented carbides. We model configurational degrees of freedom on two sublattices $(\mathrm{W}, \mathrm{Ti})$ and $(\mathrm{C}$, vacancy) using alloy cluster expansions and Monte Carlo simulations. Vibrational contributions to the interface free energy are taken into account using the harmonic approximation for various ground-state structures. We employ THERMO-CALC to find relevant chemical potentials for $\mathrm{Ti}$ and $\mathrm{C}$ as a function of composition in a relevant Ti-doped WC-Co material.

This allows us to construct an interfacial phase diagram as a function of the Ti dopant concentration, which reveals that Ti cubic films are stabilized in the material for lower dopant concentrations than for which the $\gamma-(\mathrm{Ti}, \mathrm{W}) \mathrm{C}_{x}$ bulk phase would precipitate. Further, the thin cubic films are stable both at solid-state and liquid-phase sintering temperatures. Finally, we find that Ti tends to occupy the second metal layer but not the first in the cubic films which is in perfect agreement with high-resolution transmission electron microscopy studies $[18,19]$.

\section{ACKNOWLEDGMENTS}

This project is financially supported by the Swedish Foundation for Strategic Research (Grant No. SSF: RMA 15-0062) and the Swedish Research Council (Grant No. VR: 2016-04342). The computations were enabled by resources provided by the Swedish National Infrastructure for Computing (SNIC) at NSC (Linköping) and C3SE (Göteborg) partly funded by the Swedish Research Council through Grant No. 2018-05973.

\section{APPENDIX A: ELECTRONIC STRUCTURE CALCULATIONS}

The electronic structure calculations in this study are performed using DFT as implemented in the Vienna $a b$ initio simulation package (VASP) [31,32]. The included projectoraugmented wave (PAW) [44,45] potentials and the Perdew, Burke, and Ernzerhof (PBE) exchange-correlation functional [46] are utilized. All calculations are done non-spin-polarized (for motivation see Sec. VID). Further, in all calculations the smearing method of Methfessel and Paxton to first order with a smearing width of 0.1 [47] is used and the Brillouin zone is sampled using a $\Gamma$-centered grid.

The thin-film systems, as described in Sec. II, are constructed by stacking a slab of fcc Co with 7 atomic layers in the [111] directions on top of a slab of hexagonal WC with two W-terminated (0001) surfaces and a total of 6 (5) atomic layers of W (C) in the [0001] direction. In order to end up with one phase boundary under periodic boundary conditions $10 \AA$ of vacuum is added perpendicular to the interface plane between the two remaining Co (111) and WC (0001) planes, thus creating two free surfaces. Subsequently, the stacking of $\mathrm{W}$ and $\mathrm{C}$ layers at the interface is altered to get various thin-film systems according to Fig. 1. In the parallel direction the size of the system is determined by the hexagonal WC phase. The unit cell in the interface plane is a parallelogram with base $\sqrt{3} a_{\mathrm{WC}}$ and height (3/2) $a_{\mathrm{WC}}$, where $a_{\mathrm{WC}}$ is the bulk lattice constant for $\delta$-WC (see Ref. [20] for a figure of the unit cell). Our DFT values for the bulk lattice constants are $a_{\mathrm{WC}}=2.915 \AA$ and $c_{\mathrm{WC}}=2.844 \AA$.

Further, in all VASP calculations of the thin-film systems a $2 \times 2$ supercell in the interface plane, corresponding to 244 atoms for a system without $\mathrm{C}$ vacancies, is used. The Brillouin zone is sampled with $5 k$ points along each reciprocal lattice vector in the phase boundary plane and $1 k$ point along the reciprocal lattice vector perpendicular to the interface plane, i.e., a $k$-point density of $8.04 \AA$ in the phase boundary plane. Reference calculations are done with better or equal $k$-point density.

Reference energy calculations for training the cluster expansions are done using a plane-wave cutoff of $400 \mathrm{eV}$ and evaluating the projection operators in real space, i.e., $L R E A L=$ Auto and optimize the ionic positions until all forces are below $0.02 \mathrm{eV} / \AA$. The reference data for constructing the vibrational models, which require more accurate forces, are calculated using a higher plane-wave cutoff of $520 \mathrm{eV}$ . Evaluating the projection operators in real space generally gives less accurate forces compared to evaluation in reciprocal space. However, through testing we found that evaluation in real space gives forces that are accurate enough for fitting force constants using the regression approach in HIPHIVE. This reduced the computational cost considerably. Additionally, the ionic positions are optimized until all forces are below $0.005 \mathrm{eV} / \AA$.

The major approximation within DFT theory is the exchange-correlation functional. In this paper we have used the semilocal PBE [46] exchange-correlation functional 
which has become a standard within materials science. The choice of exchange-correlation functional may effect transition temperatures and relative free energies of bulk phases by several percentages [38]. Further, absolute interface energies in WC-Co cemented carbides are also sensitive to the choice of exchange-correlation functional [48]. However, in this case the differences are usually not random but very systematic and will thus be subject to large cancellations when calculating relative interface energy with and without a thin film. Consequently, small changes in the relative interface energies and bulk energies should not drastically alter our ability to predict both the stability and order of the thin cubic films.

\section{APPENDIX B: CALPHAD MODELING}

To model the bulk phase diagram of the W-C-Co-Ti system we use the CALPHADmethodology as implemented in the software THERMO-CALC [23]. The thermodynamic data for the $\mathrm{W}-\mathrm{C}-\mathrm{Co}-\mathrm{Ti}$ system are taken from the databases TCFE9 and SSUB6 [23,24]. The same modeling technique is also used to determine the chemical potential differences $\Delta \mu_{\mathrm{C}}(T)$ and $\Delta \mu_{\mathrm{Ti}}(T)$.

The calculations are carried out for a fixed ratio between the atomic concentrations of $\mathrm{Co}$ and $\mathrm{W}$, viz., 0.373, and varying the atomic concentrations of $\mathrm{C}$ and Ti. This corresponds to a cemented carbide with a binder phase volume fraction of about 0.17 . The Co/W ratio is the same as used in Ref. [18].
[1] P. R. Cantwell, T. Frolov, T. J. Rupert, A. R. Krause, C. J. Marvel, G. S. Rohrer, J. M. Rickman, and M. P. Harmer, Annu. Rev. Mater. Res. 50, 465 (2020).

[2] H. E. Exner, Int. Mater. Rev. 24, 149 (1979).

[3] H.-O. Andrén, Mater. Des. 22, 491 (2001).

[4] J. García, V. C. Ciprés, A. Blomqvist, and B. Kaplan, Int. J. Refract. Met. Hard Mater. 80, 40 (2019).

[5] G. S. Upadhyaya, Cemented Tungsten Carbides: Production, Properties, and Testing, 1st ed. (Noyes Publications, Saddle River, NJ, 1998).

[6] Comprehensive Hard Materials, edited by V. K. Sarin (Elsevier, Oxford, 2014).

[7] L. Prakash, in Comprehensive Hard Materials, edited by V. K. Sarin (Elsevier, Oxford, 2014), p. 29.

[8] S. Norgren, J. García, A. Blomqvist, and L. Yin, Int. J. Refract. Met. Hard Mater. 48, 31 (2015).

[9] B. Roebuck, Int. J. Refract. Met. Hard Mater. 24, 101 (2006).

[10] W. D. Schubert, A. Bock, and B. Lux, Int. J. Refract. Met. Hard Mater.(Special Issue on Fine Grained Hardmetals)13, 281 (1995).

[11] Z. Z. Fang, X. Wang, T. Ryu, K. S. Hwang, and H. Sohn, Int. J. Refract. Met. Hard Mater. 27, 288 (2009), International Conference on the Science of Hard Materials-9.

[12] X. Liu, X. Song, H. Wang, X. Liu, F. Tang, and H. Lu, Acta Mater. 149, 164 (2018).

[13] S. Farag, I. Konyashin, and B. Ries, Int. J. Refract. Met. Hard Mater. 77, 12 (2018).

[14] J. W. Cahn, Acta Metall. 10, 789 (1962).

[15] M. Hillert and B. Sundman, Acta Metall. 24, 731 (1976).

[16] T. Yamamoto, Y. Ikuhara, and T. Sakuma, Sci. Technol. Adv. Mater. 1, 97 (2000)

[17] A. Delanoë, M. Bacia, E. Pauty, S. Lay, and C. H. Allibert, J. Cryst. Growth 270, 219 (2004).

[18] A. Meingast, E. Coronel, A. Blomqvist, S. Norgren, G. Wahnström, and M. Lattemann, Int. J. Refract. Met. Hard Mater. 72, 135 (2018).

[19] C. Yang, C. Hu, C. Xiang, H. Nie, X. Gu, L. Xie, J. He, W. Zhang, Z. Yu, and J. Luo, Sci. Adv. 7, eabf6667 (2021).

[20] S. A. E. Johansson and G. Wahnström, Phys. Rev. B 86, 035403 (2012).

[21] M. Ångqvist, W. A. Muñoz, J. M. Rahm, E. Fransson, C. Durniak, P. Rozyczko, T. H. Rod, and P. Erhart, Adv. Theory Simul. 2, 1900015 (2019).
[22] F. Eriksson, E. Fransson, and P. Erhart, Adv. Theory Simul. 2 , 1800184 (2019).

[23] J.-O. Andersson, T. Helander, L. Höglund, P. Shi, and B. Sundman, Calphad 26, 273 (2002).

[24] See, https://thermocalc.com/.

[25] A. S. Kurlov and A. I. Gusev, Inorg. Mater. 42, 121 (2006).

[26] E. Fransson, M. Gren, and G. Wahnström, Acta Mater. 216 117128 (2021).

[27] See Supplemental Material at http://link.aps.org/supplemental/ 10.1103/PhysRevMaterials.5.093801 for additional details regarding interface energies, selecting promising stackings and mixing sites, fitting of the alloy cluster expansion, vibrational calculations, chemical potentials at $1300 \mathrm{~K}$, and relative interface energies.

[28] A. Stukowski, Modell. Simul. Mater. Sci. Eng. 18, 015012 (2009).

[29] A. Sutton and R. Balluffi, Interfaces in Crystalline Materials, Monographs on the physics and chemistry of materials (Clarendon, Oxford, 1995).

[30] M. A. Gren, E. Fransson, and G. Wahnström, Int. J. Refract. Met. Hard Mater. 87, 105114 (2020).

[31] G. Kresse and J. Furthmüller, Phys. Rev. B 54, 11169 (1996).

[32] G. Kresse and J. Furthmüller, Comp. Mater. Sci. 6, 15 (1996).

[33] H. Lukas, S. G. Fries, and B. Sundman, Computational Thermodynamics: The Calphad Method, 1st ed. (Cambridge University, New York, 2007).

[34] D. Frenkel and B. Smit, Understanding Molecular Simulation: From Algorithms to Applications (Academic, San Diego, 2001).

[35] D. C. Wallace, Thermodynamics of Crystals (Wiley, New York, 1972).

[36] E. Fransson, F. Eriksson, and P. Erhart, npj Comput. Mater. 6 (2020).

[37] A. Togo and I. Tanaka, Scr. Mater. 108, 1 (2015).

[38] M. Gren, E. Fransson, M. Ångqvist, P. Erhart, and G. Wahnström, Phys. Rev. Materials 5, 033804 (2021).

[39] P. M. Larsen, K. W. Jacobsen, and J. Schiøtz, Phys. Rev. Lett. 120, 256101 (2018).

[40] S. A. E. Johansson and G. Wahnström, Philos. Mag. Lett. 90 599 (2010).

[41] S. A. Johansson and G. Wahnström, Curr. Opin. Solid State Mater. Sci. 20, 299 (2016).

[42] S. A. E. Johansson and G. Wahnström, Acta Mater. 59, 171 (2011). 
[43] V. Bounhoure, S. Lay, F. Charlot, A. Antoni-Zdziobek, E. Pauty, and J. Missiaen, Int. J. Refract. Met. Hard Mater. 44, 27 (2014).

[44] P. E. Blöchl, Phys. Rev. B 50, 17953 (1994).

[45] G. Kresse and D. Joubert, Phys. Rev. B 59, 1758 (1999).
[46] J. P. Perdew, K. Burke, and M. Ernzerhof, Phys. Rev. Lett. 77, 3865 (1996).

[47] M. Methfessel and A. T. Paxton, Phys. Rev. B 40, 3616 (1989).

[48] M. V. G. Petisme, S. A. E. Johansson, and G. Wahnström, Model. Simul. Mater. Sc. 23, 045001 (2015). 